\title{
On the weak amenability of $\mathcal{B}(X)$
}

by

\author{
A. Blanco (Belfast)
}

\begin{abstract}
We investigate the weak amenability of the Banach algebra $\mathcal{B}(X)$ of all bounded linear operators on a Banach space $X$. Sufficient conditions are given for weak amenability of this and other Banach operator algebras with bounded one-sided approximate identities.
\end{abstract}

1. Introduction. Let $\mathcal{A}$ be a Banach algebra and let $\mathfrak{X}$ be a Banach $\mathcal{A}$-bimodule. A (bounded) derivation is a (bounded) linear map $D: \mathcal{A} \rightarrow \mathfrak{X}$ that satisfies the identity

$$
D(a b)=D(a) \cdot b+a \cdot D(b) \quad(a, b \in \mathcal{A}) .
$$

Every map from $\mathcal{A}$ to $\mathfrak{X}$ of the form $a \mapsto a \cdot x-x \cdot a(a \in \mathcal{A})$, where $x \in \mathfrak{X}$ is fixed, is a bounded derivation. Derivations of this form are called inner. The first Hochschild-Johnson cohomology group of $\mathcal{A}$ with coefficients in an $\mathcal{A}$-bimodule $\mathfrak{X}$, denoted $\mathcal{H}^{1}(\mathcal{A}, \mathfrak{X})$, is defined to be the quotient of the space of bounded derivations from $\mathcal{A}$ to $\mathfrak{X}$ by the corresponding (sub)space of inner derivations. Thus, triviality of $\mathcal{H}^{1}(\mathcal{A}, \mathfrak{X})$ amounts to every continuous derivation from $\mathcal{A}$ into $\mathfrak{X}$ being inner.

The topological dual $\mathfrak{X}^{\prime}$ of a Banach $\mathcal{A}$-bimodule $\mathfrak{X}$ is also a Banach $\mathcal{A}$-bimodule under the actions

$$
(a \cdot f)(x)=f(x a) \quad \text { and } \quad(f \cdot a)(x)=f(a x) \quad\left(a \in \mathcal{A}, x \in \mathfrak{X}, f \in \mathfrak{X}^{\prime}\right) .
$$

In particular, $\mathcal{A}^{\prime}$ becomes a Banach $\mathcal{A}$-bimodule in this way. A Banach algebra $\mathcal{A}$ is said to be weakly amenable if $\mathcal{H}^{1}\left(\mathcal{A}, \mathcal{A}^{\prime}\right)=\{0\}$. The notion of weak amenability was introduced in [BCD] for commutative Banach algebras and extended to the general case in [J2]. For instance, group algebras of locally compact groups, $C^{*}$-algebras and tensor algebras are all examples of weakly amenable Banach algebras (see [J3], [H] and [DGG], respectively). For further examples see [Da].

2010 Mathematics Subject Classification: Primary 46B20, 47L10; Secondary 16E40. Key words and phrases: Banach algebra, Banach space, weakly amenable. 
In this paper, we shall be primarily concerned with the weak amenability of the Banach algebra $\mathcal{B}(X)$ of all bounded linear operators on a Banach space $X$. Some remarks on weak amenability of this algebra were made in DGG, where it was noticed that if $X$ is a reflexive Banach space so that $X \simeq \ell_{p}(X)$ for some $1<p<\infty$, then an argument similar to the one used in the proof of [W, Proposition 5] shows that the homology groups of $\mathcal{B}(X)$ with coefficients in itself vanish, which in turn, combined with JJ1, Corollary 1.3], implies that $\mathcal{H}^{n}\left(\mathcal{B}(X), \mathcal{B}(X)^{\prime}\right)=\{0\}(n \in \mathbb{N})$. We give below an elementary version of this argument in the case $n=1$.

In the opposite direction, one should mention that examples of Banach spaces $X$ for which $\mathcal{B}(X)$ is not weakly amenable have appeared in the literature. For instance, if $\mathcal{R}$ is the Banach space constructed in $[\mathbb{R}$ then $\mathcal{B}(\mathcal{R})$ cannot be weakly amenable as one can easily define non-zero, continuous point derivations on $\mathcal{B}(\mathcal{R})$ (see [DGG, Proposition 1.3]). Another (more tractable) example is the Banach space constructed in [B12, Proposition 5.3]. The latter happens to be a reflexive Banach space with an unconditional basis. That the algebra of bounded operators on it is not weakly amenable follows readily from [B12, Proposition 5.3] and [DGG, Theorem 5.6].

The question of when $\mathcal{B}(X)$ is weakly amenable was formally raised in $\mathrm{G}$, Question 22] and it is our view that little progress has been made in the study of this problem since [DGG]. Here, we will give sufficient conditions for weak amenability of $\mathcal{B}(X)$ and other Banach operator algebras with bounded onesided approximate identities. In the case of $\mathcal{B}(X)$, these conditions will be seen to have an easy interpretation in terms of the geometry of $X$. Moreover, they will be verified in a number of important examples.

To some extent, this work could be seen as a continuation of our earlier research on weak amenability of Banach algebras of approximable operators. Indeed, some of the ideas of this paper will be found to be reminiscences of ideas from [B]]. Essential to the results of the latter were the facts that the tensor algebra is always weakly amenable and that the continuous finite-rank operators are dense in the tensor algebra and in the algebra of approximable operators on any Banach space. The absence, in general, of a weakly amenable dense subalgebra in $\mathcal{B}(X)$ was one of the main obstacles in extending results from [B] to Banach algebras of bounded operators. In this paper, we will follow a slightly different approach which will allow us to overcome this difficulty and, consequently, to make further progress in the study of weak amenability of $\mathcal{B}(X)$. The present work will provide, in addition, a new framework in which to accommodate known results on weak amenability of algebras of approximable operators.

The organization of the paper is as follows. In the next section, we have gathered some notation and terminology that we need. The main result of 
the note is proved in Section 3. It is then applied, in Section 4, to establish the weak amenability of $\mathcal{B}(X)$ in cases where $X$ admits a relatively nice direct sum decomposition. In Section 5, we then look at cases where such a nice direct sum decomposition is not possible. The main examples considered in this section are of Tsirelson-like type. Finally, in Section 6, we turn our attention to algebras of bounded operators acting on finite direct sums of Banach spaces with $\ell_{p}$-sum decompositions.

2. Some notations and terminology. Throughout, we write $X^{\prime}$ for the topological dual of a normed space $X$, and given a subset $S$ of $X$, we denote by $[S]$ the closure of its linear span.

By a (topological) direct sum decomposition of a Banach space $X$ we mean a sequence $\left(X_{i}\right)$ of closed subspaces of $X$ such that every $x \in X$ can be represented in a unique way as the sum of a series $\sum_{i} x_{i}$, where $x_{i} \in X_{i}$ $(i \in \mathbb{N})$. We write this as $X=\bigoplus_{i=1}^{\infty} X_{i}$.

Given Banach spaces $X_{1}, \ldots, X_{n}$, we denote by $X_{1} \oplus \cdots \oplus X_{n}$ (or by $\oplus_{i=1}^{n} X_{i}$ ) the linear space $X_{1} \times \cdots \times X_{n}$ endowed (unless otherwise specified) with any norm with respect to which all canonical coordinate projections and embeddings are continuous. Also, given a Banach space $X$, we write $\ell_{p}(X), 1 \leq p \leq \infty$, (resp. $\left.c_{0}(X)\right)$ for the $\ell_{p}$-sum (resp. $c_{0}$-sum) of infinitely many copies of $X$, i.e., the linear space of all sequences $\left(x_{i}\right)$ in $X$ so that $\left(\left\|x_{i}\right\|\right) \in \ell_{p}\left(\operatorname{resp} .\left(\left\|x_{i}\right\|\right) \in c_{0}\right)$, endowed with the norm $\left\|\left(x_{i}\right)\right\|:=\left\|\left(\left\|x_{i}\right\|\right)\right\|_{p}$ (resp. $\left.\left\|\left(x_{i}\right)\right\|:=\left\|\left(\left\|x_{i}\right\|\right)\right\|_{\infty}\right)$.

If $X$ and $Y$ are isomorphic (resp. isometric) normed spaces, we write this as $X \simeq Y$ (resp. $X \cong Y)$, and denote by $d(X, Y)$ the Banach-Mazur distance between them, i.e., the infimum of numbers $\|T\|\left\|T^{-1}\right\|$, where $T: X \rightarrow Y$ is a linear isomorphism. The identity operator on a normed space $X$ is denoted by $\operatorname{id}_{X}$.

Recall that a bounded left (resp. right) approximate identity, b.l.a.i. (resp. b.r.a.i.) for short, for a Banach algebra $\mathcal{A}$ is a bounded net $\left(e_{\alpha}\right)$ in $\mathcal{A}$ with the property that $\lim _{\alpha} e_{\alpha} a=a\left(\operatorname{resp} . \lim _{\alpha} a e_{\alpha}=a\right)$ for every $a \in \mathcal{A}$.

We call a Banach $\mathcal{A}$-bimodule $\mathfrak{X}$ left essential if $\mathfrak{X}=[\mathcal{A} \mathfrak{X}]$, where $\mathcal{A} \mathfrak{X}=$ $\{a x: a \in \mathcal{A}, x \in \mathfrak{X}\}$. If $\mathcal{A}$ has a b.l.a.i. (resp. b.r.a.i.) $\left(e_{\alpha}\right)$ then, by Cohen's factorization theorem, $\mathfrak{X}$ is left (resp. right) essential if and only if $\lim _{\alpha} e_{\alpha} x=$ $x$ (resp. $\lim _{\alpha} x e_{\alpha}=x$ ) for every $x \in \mathfrak{X}$.

We denote by $\mathcal{A}(X)$ the uniform closure in $\mathcal{B}(X)$ of the ideal $\mathcal{F}(X)$ of continuous finite-rank operators on $X$, and by $\mathcal{W}(X)$, the ideal of weakly compact operators on $X$.

Lastly (though this is not essential), we assume all our normed spaces to be over the complex field. 
3. Derivations from $\mathcal{B}(X)$. In this section we present the main result of the note. We start with a proof of the known fact that if $E$ is a Banach space so that $E \simeq \ell_{p}(E)$ then every derivation from $\mathcal{B}(E)$ into $\mathcal{B}(E)^{\prime}$ is inner. As indicated in the introduction, the proof is inspired by Wodzicki's ideas $([\mathrm{W}])$ and it will serve as a motivation for our subsequent results.

We shall need the following.

Lemma 3.1. Let $\mathcal{A}$ be a Banach algebra, let $\mathfrak{X}$ be a Banach $\mathcal{A}$-bimodule and let $D: \mathcal{A} \rightarrow \mathfrak{X}^{\prime}$ be a derivation. Let $e \in \mathcal{A}$ be an idempotent and let $g, h \in \mathcal{A}$ be such that $g h=e$. Define $D_{g, h}: \mathcal{A} \rightarrow \mathfrak{X}^{\prime}$ by $D_{g, h}(a):=g \cdot D(h a g) \cdot h$ $(a \in \mathcal{A})$. Then for every $a \in e \mathcal{A} e$ and every $y \in e \mathfrak{X} e$,

$$
\langle y, D(a)\rangle-\left\langle y, D_{g, h}(a)\right\rangle=\langle y a-a y, g \cdot D(h)\rangle .
$$

Proof. Straightforward computations using the fact that $e \cdot D(e) \cdot e=0$.

Proposition 3.2. If $E$ is a Banach space so that either $E \simeq \ell_{p}(E)$ for some $1 \leq p \leq \infty$ or $E \simeq c_{0}(E)$ then $\mathcal{B}(E)$ is weakly amenable.

Proof. We give the proof only for the case where $E \simeq \ell_{p}(E)$ for some $1 \leq p \leq \infty$, the case where $E \simeq c_{0}(E)$ being completely analogous.

Let $D: \mathcal{B}(E) \rightarrow \mathcal{B}(E)^{\prime}$ be a continuous derivation and let $\phi: E \rightarrow \ell_{p}(E)$ be a Banach space isomorphism. Define $\widetilde{D}: \mathcal{B}\left(E \oplus \ell_{p}(E)\right) \rightarrow \mathcal{B}\left(E \oplus \ell_{p}(E)\right)^{\prime}$ by

$$
\begin{aligned}
\left\langle\left(\begin{array}{ll}
u_{11} & u_{12} \\
u_{21} & u_{22}
\end{array}\right), \widetilde{D}\left(\begin{array}{ll}
v_{11} & v_{12} \\
v_{21} & v_{22}
\end{array}\right)\right\rangle & \\
= & \left\langle u_{11}, D\left(v_{11}\right)\right\rangle+\left\langle u_{12} \phi, D\left(\phi^{-1} v_{21}\right)\right\rangle \\
& +\left\langle\phi^{-1} u_{21}, D\left(v_{12} \phi\right)\right\rangle+\left\langle\phi^{-1} u_{22} \phi, D\left(\phi^{-1} v_{22} \phi\right)\right\rangle .
\end{aligned}
$$

One verifies that $\widetilde{D}$ is a derivation.

Next, for every $w \in \mathcal{B}(E)$, define

$$
\Delta(w):=\left(\begin{array}{ccc}
w & & \\
& w & \\
& & \ddots
\end{array}\right) \in \mathcal{B}\left(\ell_{p}(E)\right) .
$$

Let $L$ and $R$ be left and right shifts, respectively, with respect to the direct sum decomposition $E \oplus \ell_{p}(E)$, so that $L R=\operatorname{id}_{E \oplus \ell_{p}(E)}$ and

$$
R\left(\begin{array}{ll}
w & \\
& \Delta(w)
\end{array}\right) L=\left(\begin{array}{ll}
0 & \\
& \Delta(w)
\end{array}\right) \quad(w \in \mathcal{B}(E)) .
$$


Then, by Lemma 3.1, for all $u$ and $v$ in $\mathcal{B}(E)$,

$$
\begin{aligned}
\langle u, D(v)\rangle= & \left\langle\left(\begin{array}{ll}
u & \\
& \Delta(u)
\end{array}\right), \widetilde{D}\left(\begin{array}{ll}
v & \\
& \Delta(v)
\end{array}\right)\right\rangle \\
& \left.-\left\langle\left(\begin{array}{ll}
0 & \\
& \Delta(u)
\end{array}\right), \widetilde{D}\left(\begin{array}{cc}
0 & \\
& \Delta(v)
\end{array}\right)\right)\right\rangle \\
= & \left\langle\left(\begin{array}{rr}
u v-v u & \\
& \Delta(u v-v u)
\end{array}\right), L \cdot \widetilde{D}(R)\right\rangle .
\end{aligned}
$$

Letting $T: \mathcal{B}(E) \rightarrow \mathcal{B}\left(E \oplus \ell_{p}(E)\right), v \mapsto\left({ }^{v} \Delta(v)\right)$, and $\xi=T^{\prime}(L \cdot \widetilde{D}(R))$ one readily deduces from the last identity that

$$
\langle u, D(v)\rangle=\langle u v-v u, \xi\rangle=\langle u, v \cdot \xi-\xi \cdot v\rangle \quad(u, v \in \mathcal{B}(E)),
$$

or equivalently, that $D(v)=v \cdot \xi-\xi \cdot v(v \in \mathcal{B}(E))$, as needed.

As mentioned earlier, some of the ideas behind this proof motivate most of what follows.

Recall that a unital Banach algebra $\mathcal{A}$ with identity $e$ is properly infinite if there are sequences $\left(r_{i}\right)$ and $\left(s_{i}\right)$ in $\mathcal{A}$ so that $r_{i} s_{j}=\delta_{i, j} e(i, j \in \mathbb{N})$. It is not hard to see that the proof of Proposition 3.2 can be adapted to Banach algebras with this last property, provided the sequences $\left(r_{i}\right)$ and $\left(s_{i}\right)$ are such that

(a) the "diagonal amplification" operator, $\Delta: \mathcal{A} \rightarrow \mathcal{A}, a \rightarrow \sum_{i=1}^{\infty} s_{i} a r_{i}$, is defined, i.e., $\sum_{i=1}^{\infty} s_{i} a r_{i}$ converges $(a \in \mathcal{A})$;

(b) both series, $\sum_{i=1}^{\infty} s_{i} r_{i+1}$ and $\sum_{i=1}^{\infty} s_{i+1} r_{i}$, converge.

Indeed, one can simply carry out the same argument as above, letting

$$
R=\left(\begin{array}{cc}
0 & 0 \\
s_{1} & \sum_{j} s_{j+1} r_{j}
\end{array}\right) \quad \text { and } \quad L=\left(\begin{array}{cc}
0 & r_{1} \\
0 & \sum_{j} s_{j} r_{j+1}
\end{array}\right) .
$$

For the Banach algebra $\mathcal{B}(X)$, proper infiniteness has an easy interpretation in terms of the geometry of the underlying Banach space. Indeed, it is well known (see for instance [La1, Lemma 1.8]) that $\mathcal{B}(X)$ is properly infinite if and only if $X$ admits a cartesian decomposition, i.e., if and only if $X \simeq X \oplus X \oplus Y$ for some Banach space $Y$. Unfortunately, in general, the latter is not enough to ensure that the above conditions are satisfied. An example of this situation is provided by Tsirelson's space (see Section 5). The same is probably true for other important examples. For instance, it seems unlikely that one can choose $\left(r_{i}\right)$ and $\left(s_{i}\right)$ to satisfy (a) and (b) for $X=\ell_{p} \oplus \ell_{q}(1 \leq p \neq q<\infty)$ or for every Banach space with a symmetric basis, but we do not have a proof of this. Note, though, that apart from $c_{0}$ 
and $\ell_{p}(1 \leq p<\infty)$ there are other Banach spaces with a symmetric basis for which this is possible ([R1]).

To address the above limitations, we shall relax the hypotheses of Proposition 3.2 (and in turn (a) and (b)) in two main ways. First we shall consider Banach algebras with bounded one-sided approximate identities. Second, we shall consider finite diagonal amplifications of the elements of the algebra instead of infinite ones. This last idea was already present in [G1], though it was not fully exploited there. Here, we look at it in more detail.

Let us start with the following.

Lemma 3.3. Let $\mathcal{A}$ be a Banach algebra and let $e \in \mathcal{A}$ be so that there are sequences $\left(r_{n}\right)$ and $\left(s_{n}\right)$ in $\mathcal{A}$ satisfying

$$
r_{m} s_{n}=\delta_{m, n} e \quad(n, m \in \mathbb{N}) .
$$

Let $\mathfrak{X}$ be a Banach $\mathcal{A}$-bimodule and let $D: \mathcal{A} \rightarrow \mathfrak{X}^{\prime}$ be a derivation. Then, for every $a \in \mathcal{A}$ and every $x \in \mathfrak{X}$,

$$
\langle e x, D(e a)\rangle=\left\langle a e x-x e a, \varphi_{n}\right\rangle+n^{-1}\left\langle\Delta_{n}(x), D\left(\Delta_{n}(a)\right)\right\rangle \quad(n \in \mathbb{N}),
$$

with $\varphi_{n}=n^{-1} \sum_{i=1}^{n} D\left(r_{i}\right) \cdot s_{i}, \Delta_{n}(x)=\sum_{i=1}^{n} s_{i} x r_{i}$ and $\Delta_{n}(a)=\sum_{i=1}^{n} s_{i} a r_{i}$.

Proof. Let $e,\left(r_{i}\right)$ and $\left(s_{i}\right)$ be as in the hypotheses of the lemma. Let $a \in \mathcal{A}$ and $x \in \mathfrak{X}$ be arbitrary. Then

$$
\begin{aligned}
\left\langle\Delta_{n}(x), D\right. & \left.\left(\Delta_{n}(a)\right)\right\rangle \\
& =\sum_{i=1}^{n}\left\langle s_{i} x r_{i}, D\left(s_{i} a r_{i}\right)\right\rangle \\
& =n\langle e x e, D(a)\rangle+\sum_{i=1}^{n}\left(\left\langle a e x, r_{i} \cdot D\left(s_{i}\right)\right\rangle+\left\langle x e a, D\left(r_{i}\right) \cdot s_{i}\right\rangle\right) \\
& =n\langle e x e, D(a)\rangle+n\langle a e x, D(e)\rangle+\sum_{i=1}^{n}\left\langle x e a-a e x, D\left(r_{i}\right) \cdot s_{i}\right\rangle \\
& =n\langle e x, D(e a)\rangle-n\left\langle\text { aex }-x e a, \varphi_{n}\right\rangle .
\end{aligned}
$$

The rest is clear.

Now the main result of the note reads as follows.

Theorem 3.4. Let $\mathcal{A}$ be a Banach algebra with a b.l.a.i. $\left(e_{\alpha}\right)$, let $\mathfrak{X}$ be a left essential Banach $\mathcal{A}$-bimodule, and let $D: \mathcal{A} \rightarrow \mathfrak{X}^{\prime}$ be a bounded derivation. Suppose for each $e_{\alpha}$ there are sequences $\left(r_{i, \alpha}\right)$ and $\left(s_{i, \alpha}\right)$, as in Lemma 3.3 , and suppose there is an increasing sequence of positive integers, $\left(n_{k}\right)$, so that

(i) $\sup _{k, \alpha}\left\|\varphi_{k, \alpha}\right\|<\infty$, where $\varphi_{k, \alpha}:=n_{k}^{-1} \sum_{i=1}^{n_{k}} D\left(r_{i, \alpha}\right) \cdot s_{i, \alpha}$; 
(ii) there are dense subsets $\mathfrak{X}^{\circ}$ of $\mathfrak{X}$ and $\mathcal{A}^{\circ}$ of $\mathcal{A}$ such that, for every $x \in \mathfrak{X}^{\circ}$ and $a \in \mathcal{A}^{\circ}$,

$$
\lim _{k} \varlimsup_{\alpha} n_{k}^{-1}\left\langle\Delta_{n_{k}, \alpha}(x), D\left(\Delta_{n_{k}, \alpha}(a)\right)\right\rangle=0,
$$

where $\Delta_{n_{k}, \alpha}(x):=\sum_{i=1}^{n_{k}} s_{i, \alpha} x r_{i, \alpha}$ and $\Delta_{n_{k}, \alpha}(a):=\sum_{i=1}^{n_{k}} s_{i, \alpha} a r_{i, \alpha}$.

Then $D$ is inner.

Proof. Condition (i) implies that, for each $k$, the net $\left(\varphi_{k, \alpha}\right)$ has a weak-* convergent subnet, $\left(\varphi_{k, \alpha_{j}}\right)_{j \in J}$ say $\left(J\right.$ depending on $k$ ), with weak-* limit $\Phi_{k}$ of norm $\leq M:=\sup _{k, \alpha}\left\|\varphi_{k, \alpha}\right\|$. Let $a \in \mathcal{A}^{\circ}$ and $x \in \mathfrak{X}^{\circ}$ be arbitrary. By Lemma 3.3 ,

$$
\left\langle e_{\alpha} x, D\left(e_{\alpha} a\right)\right\rangle=\left\langle a e_{\alpha} x-x e_{\alpha} a, \varphi_{k, \alpha}\right\rangle+n_{k}^{-1}\left\langle\Delta_{n_{k}, \alpha}(x), D\left(\Delta_{n_{k}, \alpha}(a)\right)\right\rangle .
$$

Replacing $\alpha$ by $\alpha_{j}$ in this last identity and taking limits with respect to $j$, one obtains

$$
\langle x, D(a)\rangle=\left\langle a x-x a, \Phi_{k}\right\rangle+n_{k}^{-1} \lim _{j}\left\langle\Delta_{n_{k}, \alpha_{j}}(x), D\left(\Delta_{n_{k}, \alpha_{j}}(a)\right)\right\rangle,
$$

where we have taken into account the fact that $\mathfrak{X}$ is left essential. Next, choose a weak-* convergent subnet $\left(\Phi_{k_{d}}\right)_{d \in D}$ of $\left(\Phi_{k}\right)$. Then, replacing $k$ by $k_{d}$ in (3) and taking limits once more, this time with respect to $d$, one arrives at

$$
\begin{aligned}
\langle x, D(a)\rangle & =\langle a x-x a, \Phi\rangle+\lim _{d} \lim _{j} n_{k_{d}}^{-1}\left\langle\Delta_{n_{k_{d}}, \alpha_{j}}(x), D\left(\Delta_{n_{k_{d}}, \alpha_{j}}(a)\right)\right\rangle \\
& =\langle a x-x a, \Phi\rangle,
\end{aligned}
$$

where $\Phi \in \mathfrak{X}^{\prime}$ denotes the weak-* limit of $\left(\Phi_{k_{d}}\right)$ and the second equality follows from condition (ii). The desired result follows readily from this last formula, since $D$ is continuous and $\mathcal{A}^{\circ}$ and $\mathfrak{X}^{\circ}$ are dense in $\mathcal{A}$ and $\mathfrak{X}$, respectively.

REMARK 3.5. The hypotheses of Theorem 3.4 can be relaxed as follows. Let $\mathcal{A}$ be a Banach algebra and let $\mathfrak{X}$ be a left essential Banach $\mathcal{A}$-bimodule. Suppose there exists a Banach algebra $\mathcal{B}$ that contains $\mathcal{A}$ as a closed subalgebra together with a net $\left(e_{\alpha}\right)$ such that $\lim _{\alpha} e_{\alpha} a=a(a \in \mathcal{A})$. Let $D: \mathcal{A} \rightarrow \mathfrak{X}^{\prime}$ be a bounded derivation which can be lifted to a bounded derivation $\widetilde{D}: \mathcal{B} \rightarrow \mathfrak{Y}^{\prime}$, where $\mathfrak{Y}$ is a Banach $\mathcal{B}$-bimodule containing $\mathfrak{X}$ as a closed subspace. Lastly, suppose for each $e_{\alpha}$ there are sequences $\left(r_{i, \alpha}\right)$ and $\left(s_{i, \alpha}\right)$ in $\mathcal{B}$ satisfying (1), and suppose there is an increasing sequence of positive integers so that conditions (i) and (ii) of Theorem 3.4 hold with $\widetilde{D}$ in place of $D$. In this situation, one can show, exactly as above, that there exists $\widetilde{\Phi} \in \mathfrak{Y}^{\prime}$ such that $\widetilde{D}(a)=a \cdot \widetilde{\Phi}-\widetilde{\Phi} \cdot a(a \in \mathcal{A})$. Then note that the restriction map $\imath^{\prime}: \mathfrak{Y}^{\prime} \rightarrow \mathfrak{X}^{\prime}$ is an $\mathcal{A}$-bimodule homomorphism, so $D(a)=a \cdot \imath^{\prime}(\widetilde{\Phi})-\imath^{\prime}(\widetilde{\Phi}) \cdot a(a \in \mathcal{A})$, i.e., $D$ is inner. In this note, we will 
not make use of this degree of generality. For this reason, we have chosen to present, as our main result, the simpler one given in Theorem 3.4 .

REMARK 3.6. There is a "right" analogue of Theorem 3.4, which can be easily obtained by replacing $\mathcal{A}$ by its opposite $\mathcal{A}^{\mathrm{op}}$ and passing from bimodules over $\mathcal{A}$ to bimodules over $\mathcal{A}^{\mathrm{op}}$, via the usual functor. We will not use the "right" version in this note, so we leave the details to the reader.

Theorem 3.4 should be compared with [Bl, Proposition 2.2]. Indeed, the main difference between these results lies in the way the averages are taken. One should point out that it was precisely in connection with the averages $n^{-1}\left\langle\Delta_{n, \alpha}(u), D\left(\Delta_{n, \alpha}(v)\right)\right\rangle(u, v \in \mathcal{A})$ that the so-called trace unbounded triples were needed in [B] , combined with the facts that the tensor algebra is always weakly amenable and that the continuous finite-rank operators are dense in both the tensor algebra and the algebra of approximable operators.

There is one other Banach space property which is defined in terms of direct sum decompositions and which, together with the cartesian decomposition property, has proved useful in the study of automatic continuity of homomorphisms from $\mathcal{B}(X)$, namely, the continued bisection property. Recall from [J, Definition 3.1] that a Banach space $X$ is said to have a continued bisection if there is a sequence $\left(E_{n}\right)$ of closed subspaces of $X$ so that $E_{1}=X$ and $E_{n} \simeq E_{n+1} \oplus E_{n+1}(n \in \mathbb{N})$. In view of its similarity with a cartesian decomposition, one might expect the existence of a continued bisection to have some positive implications on the cohomological properties of $\mathcal{B}(X)$. However, as the next example shows, the existence of a continued bisection of $X$, even a "bounded" one, i.e., one for which the projection constants of the $E_{n}$ 's are uniformly bounded, is not enough to ensure the weak amenability of $\mathcal{B}(X)$.

EXAMPLE 3.7. It was shown in [B] that if $\left.\left(p_{n}\right) \subset\right] 1,2\left[\right.$ and $\left(k_{n}\right) \subset \mathbb{N}$ are strictly increasing sequences such that

$$
k_{n}^{1 / p_{n}-1 / 2} \geq \varepsilon_{n}^{-1} \quad \text { and } \quad k_{n}^{1 / p_{n+1}-1 / 2} \leq 2
$$

for some positive sequence $\left(\varepsilon_{n}\right)$ such that $\sum_{n} \varepsilon_{n}<\infty$, then $\mathcal{A}\left(\left(\oplus_{n=1}^{\infty} \ell_{p_{n}}^{k_{n}}\right)_{2}\right)$ is not weakly amenable. The sequences $\left(p_{n}\right)$ and $\left(k_{n}\right)$ are constructed inductively and it is easy to see that one can always choose the $k_{n}$ 's to be powers of 2 . On the other hand, if the $k_{n}$ 's are chosen to be powers of 2 then $\ell_{2} \oplus\left(\oplus_{n=1}^{\infty} \ell_{p_{n}}^{k_{n}}\right)_{2}$ has a continued bisection. Noting that $\left(\oplus_{n=1}^{\infty} \ell_{p_{n}}^{k_{n}}\right)_{2} \simeq$ $\ell_{2} \oplus\left(\oplus_{n=1}^{\infty} \ell_{p_{n}}^{k_{n}}\right)_{2}$, one concludes that $\mathcal{A}\left(\ell_{2} \oplus\left(\oplus_{n=1}^{\infty} \ell_{p_{n}}^{k_{n}}\right)_{2}\right)$ cannot be weakly amenable, and in turn, by [DGG. Theorem 5.6], that $\mathcal{B}\left(\ell_{2} \oplus\left(\oplus_{n=1}^{\infty} \ell_{p_{n}}^{k_{n}}\right)_{2}\right)$ cannot be weakly amenable either.

We should recall, though, that von Neumann algebras of type $\mathrm{II}_{1}$ are weakly amenable, and that any projection in a von Neumann algebra of type 
$\mathrm{II}_{1}$ can be halved, in particular, the identity. This suggests that some positive result should hold for Banach algebras of operators acting on Banach spaces with a continued bisection and without a cartesian decomposition. However, we shall not pursue this problem any further in this paper.

4. First applications. Our first applications of Theorem 3.4 will be to algebras of bounded operators acting on Banach spaces with relatively nice direct sum decompositions. Let us start by fixing some terminology.

A direct sum decomposition $\bigoplus_{i} X_{i}$ of a Banach space $X$ will be called $C$-unconditional if $\left\|\sum_{i} \varepsilon_{i} x_{i}\right\| \leq C\left\|\sum_{i} x_{i}\right\|$ for every sequence $\left(x_{i}\right) \in \prod_{i} X_{i}$ so that $\sum_{i} x_{i}$ converges and for every sequence $\left(\varepsilon_{i}\right)$ in $\mathbb{T}:=\{z \in \mathbb{C}:|z|=1\}$.

Given a Banach space $X$ with direct sum decomposition $\bigoplus_{i} X_{i}$, we shall say that $\bigoplus_{i} X_{i}$ satisfies a lower (resp. upper) r-estimate $(1 \leq r<\infty)$ if there exists a constant $c$ (resp. $C$ ) so that $\left(\sum_{i}\left\|x_{i}\right\|^{r}\right)^{1 / r} \leq c\left\|\sum_{i} x_{i}\right\|$ (resp. $\left.\left\|\sum_{i} x_{i}\right\| \leq C\left(\sum_{i}\left\|x_{i}\right\|^{r}\right)^{1 / r}\right)$ for every eventually zero sequence in $\prod_{i} X_{i}$. Furthermore, we shall say that $\bigoplus_{i} X_{i}$ satisfies a lower (resp. upper) $\infty$-estimate if there is a constant $c$ (resp. $C$ ) so that $\sup _{i}\left\|x_{i}\right\| \leq c\left\|\sum_{i} x_{i}\right\|$ (resp. $\left.\left\|\sum_{i} x_{i}\right\| \leq C \sup _{i}\left\|x_{i}\right\|\right)$ for every eventually zero sequence in $\prod_{i} X_{i}$. Clearly, every direct sum decomposition satisfies a lower $\infty$-estimate and an upper 1-estimate.

EXAMPle 4.1. Every uniformly convex Banach space $X$ with a subsymmetric basis has an unconditional direct sum decomposition $\bigoplus_{i} X_{i}$ satisfying an upper (resp. a lower) $r$-estimate, $1<r<\infty$, and such that $\sup _{i} d\left(X_{i}, X\right)<\infty$. Indeed, let $\left(e_{k}\right)$ be a subsymmetric basis for $X$, and let $\left\{N_{1}, N_{2}, \ldots\right\}$ be an infinite partition of $\mathbb{N}$ into infinite subsets. Then $\bigoplus_{i=1}^{\infty} X_{i}$, where $X_{i}=\left[e_{k}: k \in N_{i}\right](i \in \mathbb{N})$, is an unconditional direct sum decomposition for $X$. If $x_{1}, \ldots, x_{n} \in X$ are such that $x_{i} \in X_{i}(1 \leq i \leq n)$ then one can show, exactly as in the proof of Gurariüs' theorem, given in [D, Chapter VIII], that there exists $r>1$, depending only on the basis constant, and $C=C(r)>0$ so that $\left\|\sum_{i} x_{i}\right\| \leq C\left(\sum_{i}\left\|x_{i}\right\|^{r}\right)^{1 / r}$. The existence of a lower estimate is established similarly.

As a first consequence of the results from the previous section we have the following.

Corollary 4.2. Let $X$ be a Banach space with a direct sum decomposition $\bigoplus_{i=0}^{\infty} X_{i}$ satisfying a lower $p$-estimate for some $p<\infty$ (resp. an upper q-estimate for some $q>1$ ) and such that $\sup _{i \geq 1} d\left(X_{i}, X\right)<\infty$. Let $\pi_{i} \in \mathcal{B}(X)$ be the $i$-th coordinate projection corresponding to this decomposition, and let $\left(r_{i}\right)$ and $\left(s_{i}\right)$ be bounded sequences in $\mathcal{B}(X)$ such that $r_{i} s_{i}=\mathrm{id}_{X}$ and $s_{i} r_{i}=\pi_{i}(i \in \mathbb{N})$. Then $\mathcal{B}(X)$ is weakly amenable if and only if for every pair $u, v$ in a dense subset of $\mathcal{B}(X)$ and for every continuous derivation 
$D: \mathcal{B}(X) \rightarrow \mathcal{B}(X)^{\prime}$ one has

$$
n^{-1}\left\langle\Delta_{n}(u), D\left(\Delta_{n}(v)\right)\right\rangle \rightarrow 0,
$$

where $\Delta_{n}(u):=\sum_{i=1}^{n} s_{i} u r_{i}(u \in \mathcal{B}(X))$.

In proving the corollary we will make use of the following.

Lemma 4.3. Let $X$ be a Banach space with a direct sum decomposition $\bigoplus_{i=0}^{\infty} X_{i}$ satisfying a lower p-estimate and an upper q-estimate, and such that $X_{i} \simeq X(i \in \mathbb{N})$. Let $\left(r_{i}\right)$ and $\left(s_{i}\right)$ be sequences in $\mathcal{B}(X)$ so that, for every $i \in \mathbb{N}, r_{i} s_{i}=\operatorname{id}_{X}$ and $s_{i} r_{i}$ is the natural projection onto $X_{i}$ associated with the decomposition $\bigoplus_{i=0}^{\infty} X_{i}$. Then there exists $M>0$ such that

$$
\left\|\sum_{i=1}^{n} s_{i} u r_{i}\right\| \leq M n^{1 / q-1 / p}\left(\max _{1 \leq i \leq n}\left\|r_{i}\right\|\left\|s_{i}\right\|\right)\|u\| \quad(u \in \mathcal{B}(X), n \in \mathbb{N}) .
$$

Proof. We give the proof in the case where $p<\infty$. The cases where $p=\infty$ and $1 \leq q<\infty$, or $p=\infty$ and $q=\infty$, are treated in a similar way.

By hypothesis, there exist constants $c$ and $C$ such that

$$
c^{-1}\left(\sum_{i}\left\|x_{i}\right\|^{p}\right)^{1 / p} \leq\left\|\sum_{i} x_{i}\right\| \leq C\left(\sum_{i}\left\|x_{i}\right\|^{q}\right)^{1 / q},
$$

for every eventually zero sequence $\left(x_{i}\right) \in \prod_{i=0}^{\infty} X_{i}$. Let $u \in \mathcal{B}(X)$ and $x \in X$ be arbitrary, and let $x_{i}=s_{i} r_{i} x(i \in \mathbb{N})$. Then,

$$
\begin{aligned}
\left\|\sum_{i=1}^{n} s_{i} u r_{i} x\right\| & =\left\|\sum_{i=1}^{n} s_{i} u r_{i} x_{i}\right\| \leq C\left(\sum_{i=1}^{n}\left\|s_{i} u r_{i}\right\|^{q}\left\|x_{i}\right\|^{q}\right)^{1 / q} \\
& \leq C\left(\max _{1 \leq i \leq n}\left\|r_{i}\right\|\left\|s_{i}\right\|\right)\left(\sum_{i=1}^{n}\left\|x_{i}\right\|^{q}\right)^{1 / q}\|u\| \\
& \leq C\left(\max _{1 \leq i \leq n}\left\|r_{i}\right\|\left\|s_{i}\right\|\right) n^{1 / q-1 / p}\left(\sum_{i=1}^{n}\left\|x_{i}\right\|^{p}\right)^{1 / p}\|u\| \\
& \leq c C n^{1 / q-1 / p}\left(\max _{1 \leq i \leq n}\left\|r_{i}\right\|\left\|s_{i}\right\|\right)\left\|\sum_{i=1}^{n} x_{i}\right\|\|u\|,
\end{aligned}
$$

from which we conclude the desired inequality with $M=c C$.

Proof of Corollary 4.2. Let $\mathcal{B}(X)$ be weakly amenable and let $D: \mathcal{B}(X)$ $\rightarrow \mathcal{B}(X)^{\prime}$ be a continuous derivation, so there exists $\phi \in \mathcal{B}(X)^{\prime}$ such that $\langle u, D(v)\rangle=\langle u, v \cdot \phi-\phi \cdot v\rangle$ for every pair $u, v \in \mathcal{B}(X)$. Then

$$
\left\langle\Delta_{n}(u), D\left(\Delta_{n}(v)\right)\right\rangle=\left\langle\sum_{i=1}^{n} s_{i}(u v-v u) r_{i}, \phi\right\rangle \quad(u, v \in \mathcal{B}(X)) .
$$

By the lemma, the right hand side of this last identity is $o(n)$, whence the desired result. 
The opposite implication is an immediate consequence of Theorem 3.4 .

REMARK 4.4. In the last sentence of Corollary 4.2 one can replace $\mathcal{B}(X)$ by any self-induced closed two-sided ideal $\mathcal{I}$ of $\mathcal{B}(X)$. Indeed, the proof that weak amenability of $\mathcal{I}$ implies (5) is the same. As for the opposite implication, one just needs to note that since $\mathcal{I}$ is self-induced, every bounded derivation $D: \mathcal{I} \rightarrow \mathcal{I}^{\prime}$ can be extended to a bounded derivation $\widetilde{D}: \mathcal{B}(X) \rightarrow \mathcal{I}^{\prime}$ (see [BG, Lemma 2.1]). One can then apply Remark 3.5 .

REMARK 4.5. If $\mathcal{A}$ is a Banach algebra with a b.l.a.i. $\left(e_{\alpha}\right)$, and $\left(r_{i, \alpha}\right)$ and $\left(s_{i, \alpha}\right)$ are sequences as in Theorem 3.4. so that $\sup _{i, \alpha}\left\|r_{i, \alpha}\right\|\left\|s_{i, \alpha}\right\|<\infty$ and for each $\alpha$ the decomposition $\bigoplus_{i=1}^{\infty} \pi_{i, \alpha}(X)$ satisfies a lower $p$-estimate (resp. an upper $q$-estimate) for some $p<\infty$ (resp. $q>1$ ) fixed, then a conclusion similar to that of Corollary 4.2 holds. Namely, $\mathcal{A}$ is weakly amenable if and only if for every pair $u, v$ in a dense subset of $\mathcal{A}$ and for every bounded derivation $D: \mathcal{A} \rightarrow \mathcal{A}^{\prime}$ one has $\lim _{n} \varlimsup_{\alpha} n^{-1}\left\langle\Delta_{n, \alpha}(u), D\left(\Delta_{n, \alpha}(v)\right)\right\rangle=0$. The proof of this goes along the same lines as that of Corollary 4.2, so we leave the details to the reader.

To what extent is the estimate provided by Lemma 4.3 a sharp one? For instance, if $X=\ell_{p} \oplus \ell_{q}$, with $1 \leq q<p<\infty$, it is easy to produce a direct sum decomposition of $X$ satisfying a lower $p$-estimate and an upper $q$-estimate. Indeed, simply take a partition $\left\{N_{i}: i \in \mathbb{N}\right\}$ of $\mathbb{N}$ in which each $N_{i}$ is an infinite subset, and set $X_{i}=\left\{x \in \ell_{p}: \operatorname{supp} x \subseteq N_{i}\right\}$ and $Y_{i}=\left\{y \in \ell_{q}\right.$ : $\left.\operatorname{supp} y \subseteq N_{i}\right\}(i \in \mathbb{N})$. Then $\bigoplus_{i=1}^{\infty}\left(X_{i} \oplus Y_{i}\right)$ is a decomposition of $\ell_{p} \oplus \ell_{q}$ with the required properties. One easily verifies that, with respect to this decomposition, the order of growth of $\left\|\Delta_{n}\right\|$ is at most $n^{1 / q-1 / p}$. However, we do not know whether this estimate is best possible. An interesting fact that one should notice is that, in this case, modulo the compact operators, $\left(\Delta_{n}\right)$ is a bounded sequence.

The following is an immediate consequence of Corollary 4.2 .

Corollary 4.6. Let $X$ be a Banach space with a subsymmetric basis $\left(e_{i}\right)$, and suppose $N_{1}, N_{2}, \ldots$ is an infinite partition of $\mathbb{N}$ into infinite subsets, so that $\bigoplus_{i}\left[e_{j}: j \in N_{i}\right]$ satisfies a lower $p$-estimate and an upper q-estimate. If $1 / q-1 / p<1 / 2$ then $\mathcal{B}(X)$ is weakly amenable.

Proof. Let $\left(N_{i}\right)$ be as in the hypotheses, so $\bigoplus_{i}\left[e_{j}: j \in N_{i}\right]$ satisfies a lower $p$-estimate and an upper $q$-estimate. By Lemma 4.3 . $\left\|\Delta_{n}(u)\right\| \leq$ $K n^{1 / q-1 / p}\|u\|(u \in \mathcal{B}(X))$ for some constant $K$ independent of $n$, and so $\left\langle\Delta_{n}(u), D\left(\Delta_{n}(v)\right)\right\rangle=o(n)$ for every bounded derivation $D: \mathcal{B}(X) \rightarrow \mathcal{B}(X)^{\prime}$. By Corollary $4.2, \mathcal{B}(x)$ is weakly amenable.

The hypotheses of Corollary 4.6 seem to be very restrictive. However, at the moment, we have no evidence in support of this. It would help to know 
whether there are Banach spaces $X$ with a symmetric basis for which $\mathcal{B}(X)$ is not weakly amenable.

Regarding derivations into general dual Banach bimodules we have the following.

Corollary 4.7. Let $X$ be a Banach space with a direct sum decomposition as in Corollary 4.2, and let $\mathfrak{X}$ be a super-reflexive left essential Banach $\mathcal{B}(X)$-bimodule. Furthermore, let $\left(r_{i}\right)$ and $\left(s_{i}\right)$ also be as in Corollary 4.2 . Then a continuous derivation $D: \mathcal{B}(X) \rightarrow \mathfrak{X}^{\prime}$ is inner if and only if for every $u$ in a dense subset of $\mathcal{B}(X)$ and every $x$ in a dense subset of $\mathfrak{X}$ one has

$$
n^{-1}\left\langle\Delta_{n}(x), D\left(\Delta_{n}(u)\right)\right\rangle \rightarrow 0 .
$$

Proof. Let $\phi \in \mathfrak{X}^{\prime}$ be arbitrary and let $D: \mathcal{B}(X) \rightarrow \mathfrak{X}^{\prime}, u \mapsto u \cdot \phi-\phi \cdot u$. We know that

$$
n^{-1}\left\langle\Delta_{n}(x), D\left(\Delta_{n}(u)\right)\right\rangle=n^{-1}\left\langle\Delta_{n}(x u-u x), \phi\right\rangle \quad(x \in \mathfrak{X}, u \in \mathcal{B}(X)) .
$$

Suppose $x u \neq u x$ and set $x_{i}=s_{i}(x u-u x) r_{i}(i \in \mathbb{N})$. Without loss of generality, we can assume $\|v y\| \leq\|v\|\|y\|$ and $\|y v\| \leq\|y\|\|v\|(y \in \mathfrak{X}, v \in$ $\mathcal{B}(X))$. Then, for any scalar sequence $\left(\alpha_{i}\right)$ and every pair $k, n \in \mathbb{N}$,

$$
\left\|\sum_{i=1}^{n} \alpha_{i} x_{i}\right\|=\left\|\left(\sum_{j=1}^{n} s_{j} r_{j}\right)\left(\sum_{i=1}^{n+k} \alpha_{i} x_{i}\right)\right\| \leq C\left\|\left(\sum_{i=1}^{n+k} \alpha_{i} x_{i}\right)\right\|,
$$

where $C$ is the constant of the decomposition, i.e., $C=\sup _{n}\left\|\sum_{j=1}^{n} \pi_{j}\right\|$. Moreover, if $M=\sup _{i}\left\|r_{i}\right\|\left\|s_{i}\right\|$ then, for every $i \in \mathbb{N}$, we have

$$
0<M^{-1}\|x u-u x\|=M^{-1}\left\|r_{i} s_{i}(x u-u x) r_{i} s_{i}\right\| \leq\left\|x_{i}\right\| \leq M\|x u-u x\| .
$$

Thus, $\left(x_{i}\right)$ is a seminormalized basic sequence in $\mathfrak{X}$, i.e., a basic sequence so that $0<\inf \left\|x_{i}\right\| \leq \sup \left\|x_{i}\right\|<\infty$. If we let $\xi_{i}=x_{i} /\left\|x_{i}\right\|(i \in \mathbb{N})$ then, by [Be, Part 4, Chapter II, Theorem 1], $\left\|\sum_{i=1}^{n} x_{i}\right\|=\left\|\sum_{i=1}^{n}\right\| x_{i}\left\|\xi_{i}\right\|=o(n)$. Clearly, this is also true if $x u=u x$, so $n^{-1}\left\langle\Delta_{n}(x u-u x), \phi\right\rangle \rightarrow 0$ as $n \rightarrow \infty$.

The opposite implication follows immediately from Theorem 3.4 .

We notice that all is needed for the above argument to work is that the basic sequence $\left(x_{i}\right)$ should satisfy $\left\|\sum_{i=1}^{n} x_{i}\right\|=o(n)$. Bearing this in mind one obtains the following variation of Corollary 4.7 .

Corollary 4.8. Let $X$ be a Banach space with an unconditional direct sum decomposition $\bigoplus_{i=0}^{\infty} X_{i}$ such that $\sup _{i>1} d\left(X_{i}, X\right)<\infty$. Let $\left(r_{i}\right)$ and $\left(s_{i}\right)$ be as in Corollary 4.2, and suppose $\sum_{i} s_{i} r_{i+1}$ and $\sum_{i} s_{i+1} r_{i}$ are well defined and power bounded. Let $\mathfrak{X}$ be a left essential Banach $\mathcal{B}(X)$-bimodule containing no isomorphic copies of $\ell_{1}$. Then a continuous derivation $D$ : $\mathcal{B}(X) \rightarrow \mathfrak{X}^{\prime}$ is inner if and only if for every $u$ in a dense subset of $\mathcal{B}(X)$ and every $x$ in a dense subset of $\mathfrak{X}$ one has 


$$
n^{-1}\left\langle\Delta_{n}(x), D\left(\Delta_{n}(u)\right)\right\rangle \rightarrow 0 .
$$

Thus, in particular, every continuous derivation from $\mathcal{B}\left(\ell_{p}\right)$ into a left essential $\mathcal{B}\left(\ell_{p}\right)$-bimodule with no subspaces isomorphic to $\ell_{1}$ is inner.

Proof. The proof is almost the same as that of Corollary 4.7. One just needs to note that under the present hypotheses, the seminormalized basic sequence $\left(x_{i}\right)$, defined as in the proof of Corollary 4.7, is unconditional and the left and right shifts with respect to it are power bounded. Indeed, for every sequence $\left(\alpha_{i}\right)$ in $\mathbb{C}$, every sequence $\left(\varepsilon_{i}\right)$ in $\mathbb{T}$ and every $n \in \mathbb{N}$ one has

$$
\left\|\sum_{i=1}^{n} \varepsilon_{i} \alpha_{i} x_{i}\right\|=\left\|\left(\sum_{j} \varepsilon_{j} s_{j} r_{j}\right)\left(\sum_{i=1}^{n} \alpha_{i} x_{i}\right)\right\| \leq K\left\|\sum_{i=1}^{n} \alpha_{i} x_{i}\right\|,
$$

where $K$ is the unconditional constant of the decomposition, i.e., $K=$ $\sup _{\varepsilon}\left\|\sum_{j} \varepsilon_{j} \pi_{j}\right\|$. So, $\left(x_{i}\right)$ is an unconditional basis. As for the left and right shifts with respect to $\left(x_{i}\right)$ being power bounded, note that if $L:\left[x_{i}\right] \rightarrow\left[x_{i}\right]$, $\sum_{i} \alpha_{i} x_{i} \mapsto \sum_{i} \alpha_{i+1} x_{i}$ and $R:\left[x_{i}\right] \rightarrow\left[x_{i}\right], \sum_{i} \alpha_{i} x_{i} \mapsto \sum_{i} \alpha_{i} x_{i+1}$ then, for every $n \in \mathbb{N}$,

$$
\begin{aligned}
\left\|L^{n}\left(\sum_{i} \alpha_{i} x_{i}\right)\right\| & =\left\|\left(\sum_{j} s_{j} r_{j+n}\right)\left(\sum_{i} \alpha_{i} x_{i}\right)\left(\sum_{k} s_{k+n} r_{k}\right)\right\| \\
& =\left\|\left(\sum_{j} s_{j} r_{j+1}\right)^{n}\left(\sum_{i} \alpha_{i} x_{i}\right)\left(\sum_{k} s_{k+1} r_{k}\right)^{n}\right\| \leq N\left\|\sum_{i} \alpha_{i} x_{i}\right\|,
\end{aligned}
$$

where $N=\sup _{n}\left\|\left(\sum_{j} s_{j} r_{j+1}\right)^{n}\right\|\left\|\left(\sum_{k} s_{k+1} r_{k}\right)^{n}\right\|$, and likewise,

$$
\left\|R^{n}\left(\sum_{i} \alpha_{i} x_{i}\right)\right\| \leq\left\|\left(\sum_{j} s_{j+1} r_{j}\right)^{n}\left(\sum_{i} \alpha_{i} x_{i}\right)\left(\sum_{k} s_{k} r_{k+1}\right)^{n}\right\| \leq N\left\|\sum_{i} \alpha_{i} x_{i}\right\| .
$$

Next note that if there were a constant $c>0$ so that $\left\|\sum_{i=1}^{n} x_{i}\right\| \geq c n$ for infinitely many values of $n$, then for such $n$ 's and for every scalar sequence $\left(\alpha_{i}\right)$ one would have, letting $\sigma$ be the cyclic permutation $(12 \ldots n)$,

$$
\begin{aligned}
c \sum_{i=1}^{n}\left|\alpha_{i}\right| & \leq n^{-1}\left\|\sum_{i=1}^{n}\left(\sum_{k=1}^{n}\left|\alpha_{\sigma^{k}(i)}\right|\right) x_{i}\right\| \\
& \leq n^{-1} \sum_{k=1}^{n}\left(\left\|\sum_{i=1}^{n-k}\left|\alpha_{\sigma^{k}(i)}\right| x_{i}\right\|+\left\|\sum_{i=n-k+1}^{n}\left|\alpha_{\sigma^{k}(i)}\right| x_{i}\right\|\right) \\
& \leq n^{-1} \sum_{k=1}^{n}\left(\left\|L^{k}\right\|(1+C)\left\|\sum_{i=1}^{n}\left|\alpha_{i}\right| x_{i}\right\|+\left\|R^{n-k}\right\| C\left\|\sum_{i=1}^{n}\left|\alpha_{i}\right| x_{i}\right\|\right) \\
& \leq M\left\|\sum_{i=1}^{n} \alpha_{i} x_{i}\right\| \leq M\left(\sup _{i}\left\|x_{i}\right\|\right) \sum_{i=1}^{n}\left|\alpha_{i}\right|,
\end{aligned}
$$

where $C$ denotes the basis constant of $\left(x_{i}\right)$ and $M=(1+2 C) K N$. 
To finish the proof, simply note that, as no subspace of $\mathfrak{X}$ is isomorphic to $\ell_{1}$, we must have $\left\|\sum_{i=1}^{n} x_{i}\right\|=o(n)$.

REMARK 4.9. Note that $\sum_{i} s_{i} r_{i+1}$ and $\sum_{i} s_{i+1} r_{i}$, in the statement of Corollary 4.8 , can be seen as left and right shifts operators, respectively, with respect to the decomposition $\bigoplus_{i=1}^{\infty} X_{i}$ of $X$.

Of course, there are also analogues of Corollaries 4.7 and 4.8 for right essential bimodules. In this respect, see Remark 3.6 above.

Our next example, the James space, comes to illustrate the fact that the applications of Theorem 3.4 are not restricted to the setting of Banach spaces with a cartesian decomposition. Though we will not need its definition, let us recall that the James space, $\mathfrak{J}$, is defined to be the completion of the linear space of all complex sequences with finite support in the norm

$$
\begin{aligned}
\left\|\left(\alpha_{n}\right)\right\|_{\mathfrak{J}}=\sup \left\{\left(\sum_{n=1}^{m-1}\left|\alpha_{i_{n}}-\alpha_{i_{n+1}}\right|^{2}\right)^{1 / 2}:\right. & m, i_{1}, \ldots, i_{m} \in \mathbb{N}, \\
& \left.m \geq 2 \text { and } i_{1}<\cdots<i_{m}\right\} .
\end{aligned}
$$

It is well known that $\mathfrak{J}$ does not admit a cartesian decomposition; however, as another consequence of Theorem 3.4 we have the following.

COROLlary 4.10. The Banach algebras $\mathcal{W}(\mathfrak{J})$ and $\mathcal{B}(\mathfrak{J})$ are weakly amenable.

Proof. Let $\left(e_{\gamma}\right)$ be a bounded left approximate identity for $\mathcal{W}(\mathfrak{J})$ (see OT, Proposition 2.5]). We show next that for each element $e_{\gamma}$ there are sequences $\left(r_{i, \gamma}\right)$ and $\left(s_{i, \gamma}\right)$ in $\mathcal{W}(\mathfrak{J})$ such that the conditions of Theorem 3.4 are satisfied.

Let $\left(x_{i}\right)$ be the unit vector basis of $\mathfrak{J}$, let $J:=\left(\bigoplus_{n=1}^{\infty}\left[x_{i}\right]_{1}^{n}\right)_{\ell_{2}}$ and let $\mathcal{G}_{J}$ be the operator ideal of all bounded linear operators that factor through $J$, with the usual operator-ideal norm. It is well known that $\mathfrak{J} \simeq \mathfrak{J} \oplus \ell_{2}(J)([\mathrm{Bl}$, Lemma 3.9]) and that $\mathcal{W}(\mathfrak{J})=\mathcal{G}_{J}(\mathfrak{J})([\mathrm{La}$, Theorem 4.3]). Thus, there is $\kappa>0$ such that for every $w \in \mathcal{W}(\mathfrak{J})$ there are bounded operators, $v: \mathfrak{J} \rightarrow J$ and $u: J \rightarrow \mathfrak{J}$, satisfying $u v=w$ and $\|u\|\|v\| \leq \kappa\|w\|$. In particular, for every $e_{\gamma}$ there are linear operators $r_{\gamma}: J \rightarrow \mathfrak{J}$ and $s_{\gamma}: \mathfrak{J} \rightarrow J$ so that $r_{\gamma} s_{\gamma}=e_{\gamma}$ and $\left\|r_{\gamma}\right\|\left\|s_{\gamma}\right\| \leq \kappa\left\|e_{\gamma}\right\|$. Let $\pi_{n}: \mathfrak{J} \oplus \ell_{2}(J) \rightarrow J$ (resp. $\imath_{n}:$ $\left.J \rightarrow \mathfrak{J} \oplus \ell_{2}(J)\right)$ be the natural projection onto the $n$th summand of $\ell_{2}(J)$ (resp. the natural inclusion of the $n$th summand of $\ell_{2}(J)$ into $\mathfrak{J} \oplus \ell_{2}(J)$ ), and let $\phi: \mathfrak{J} \rightarrow \mathfrak{J} \oplus \ell_{2}(J)$ be a Banach space isomorphism. Define $s_{n, \gamma}:=\phi^{-1} \imath_{n} s_{\gamma}$ and $r_{n, \gamma}:=r_{\gamma} \pi_{n} \phi(n \in \mathbb{N})$. One easily verifies that the sequences $\left(s_{n, \gamma}\right)$ and $\left(r_{n, \gamma}\right)$ satisfy the conditions of Theorem 3.4 for every bounded derivation $D: \mathcal{W}(\mathfrak{J}) \rightarrow \mathcal{W}(\mathfrak{J})^{\prime}$, so $\mathcal{W}(\mathfrak{J})$ is weakly amenable.

That $\mathcal{B}(\mathfrak{J})$ is also weakly amenable now follows easily from the result of the previous paragraph, the well known fact that $\mathcal{B}(\mathfrak{J}) \simeq \mathcal{W}(\mathfrak{J}) \oplus \mathbb{C}$ and 
the fact that the unitization of a weakly amenable Banach algebra is weakly amenable ([DGG, Proposition 1.4(ii)]).

Let us say that a Banach space $X$ has the factorization-norm property if for every pair $(Y, Z)$ of Banach spaces, the function $\gamma_{X}: \mathcal{F}(Y, Z) \rightarrow \mathbb{R}_{+}$ defined by

$$
\gamma_{X}(T: Y \rightarrow Z):=\inf \{\|R\|\|S\|: R S=T, S: Y \rightarrow X \text { and } R: X \rightarrow Z\}
$$

is a norm on $\mathcal{F}(Y, Z)$, the space of all continuous finite-rank operators from $Y$ to $Z$ (see [BG1, Section 4]). Now the last corollary can be generalized as follows.

Corollary 4.11. Let $X$ be a Banach space with the factorization-norm property and let $Y$ be a Banach space containing a complemented subspace isomorphic either to $\ell_{p}(X)$ for some $1 \leq p<\infty$ or to $c_{0}(X)$. If $\mathcal{G}_{X}(Y)$ has a b.l.a.i. (or a b.r.a.i.) then $\mathcal{G}_{X}(Y)$ is weakly amenable.

Proof. Except for some obvious modifications the proof is almost the same as that of Corollary 4.10 .

Recall that a Banach space $X$ is said to be an $\mathcal{L}_{p^{-}}$space if it contains a net $\left(X_{\alpha}\right)$ of finite-dimensional subspaces, directed by inclusion, whose union is dense in $X$, and such that $\sup _{\alpha} d\left(X_{\alpha}, \ell_{p}^{\operatorname{dim} X_{\alpha}}\right)<\infty([\mathrm{LP}])$. We do not know whether or not $\mathcal{B}(X)$ is weakly amenable for every $\mathcal{L}_{p}$-space $X$. Of course, it is weakly amenable whenever $X$ admits a decomposition as in Corollary 4.2 , which happens to be the case for all isomorphism types of $\mathcal{L}_{p}$-spaces that we have knowledge of.

5. On Tsirelson-like spaces. In many important examples of Banach spaces, the existence of a cartesian decomposition leads to the existence of a topological direct sum decomposition $\bigoplus_{i=0}^{\infty} X_{i}$ with the property that $\sup _{i} d\left(X_{i}, X\right)<\infty$. This, however, does not seem to be always the case. For instance, if $T$ is the dual of Tsirelson's space and $K \geq 1$ then there are no bounded sequences $\left(r_{i}\right)$ and $\left(s_{i}\right)$ in $\mathcal{B}(T)$ such that $r_{i} s_{j}=\delta_{i, j} \operatorname{id}_{T}(i, j \in \mathbb{N})$ and

$$
\left\|s_{i} r_{i}(x)\right\| \leq K\left\|\left(s_{i} r_{i}+s_{j} r_{j}\right)(x)\right\| \quad(i, j \in \mathbb{N}, i<j, x \in T) .
$$

The proof of this is the same as that of [CS, Prop. X.c.2]. Unfortunately, the latter reference contains a few typos, so we have decided to include the proof here. Let us start by recalling the definition of $T$.

Given finite subsets $E$ and $F$ of $\mathbb{N}$, let us write $E<F$ if $\max E<\min F$. Let $c_{00}$ denote, as customary, the space of all scalar sequences with finite support, and let $\left(t_{n}\right)$ be the unit vector basis of $c_{00}$. Given $E \subseteq \mathbb{N}$ and $x=\sum_{n} \alpha_{n} t_{n} \in c_{00}$, let $E x=\sum_{n \in E} \alpha_{n} t_{n}$. Set $\|\cdot\|_{0}:=\|\cdot\|_{c_{0}}$ and for every 
$m \geq 0$ define

$$
\|x\|_{m+1}:=\max \left\{\|x\|_{m}, 2^{-1} \max \left[\sum_{j=1}^{k}\left\|E_{j} x\right\|_{m}\right]\right\} \quad\left(x \in c_{00}\right),
$$

where the inner maximum is taken over all possible choices of finite subsets $E_{1}, \ldots, E_{k}$ of $\mathbb{N}$ so that $\{k\} \leq E_{1}<\cdots<E_{k}$. It can be shown that $\|x\|:=$ $\lim _{m}\|x\|_{m}\left(x \in c_{00}\right)$ defines a norm on $c_{00}$. The dual $T$ of Tsirelson's space is then defined to be the completion of $c_{00}$ with respect to this last norm.

Now, to establish the claim, suppose towards a contradiction that there are bounded sequences $\left(r_{i}\right)$ and $\left(s_{i}\right)$ in $\mathcal{B}(T)$ with the required properties. Let $\delta=1 /(4 K)$, choose $m$ so that $\|L\|\left\|L^{-1}\right\|>\delta^{-1} \sup _{n}\left\|r_{n}\right\|\left\|s_{n}\right\|$ for every linear operator $L:\left[t_{i}\right]_{i=1}^{m} \rightarrow\left[t_{i}\right]_{i>m}$, and choose $n$ big enough so that whenever $x_{1}, \ldots, x_{n}$ belong to the unit ball of $\left[t_{i}\right]_{i=1}^{m}$, there exist $1 \leq$ $i, j \leq n$ such that $\left\|x_{i}-x_{j}\right\|<\delta$. Then, for some $1 \leq j_{0} \leq n$, we must have $\left\|\left(\operatorname{id}_{T}-P_{m}\right) x\right\| \geq \delta\|x\|\left(x \in X_{j_{0}}:=s_{j_{0}} P_{m}(T)\right)$, where $P_{m}$ denotes the natural projection from $T$ onto $\left[t_{i}\right]_{i=1}^{m}$. Indeed, if this were not true then for each $1 \leq j \leq n$ there would be a norm-1 vector $x_{j} \in X_{j}\left(=s_{j} P_{m}(T)\right)$ so that $\left\|\left(\operatorname{id}_{T}-P_{m}\right) x_{j}\right\|<\delta$. By our choice of $n$, for some $1 \leq i<j \leq n$ we would have $\left\|P_{m} x_{i}-P_{m} x_{j}\right\|<\delta$, and in turn, from this last and (6),

$$
\begin{aligned}
K^{-1} & =K^{-1}\left\|\left(s_{i} r_{i}\right)\left(x_{i}-x_{j}\right)\right\|=\left\|\left(s_{i} r_{i}+s_{j} r_{j}\right)\left(x_{i}-x_{j}\right)\right\|=\left\|x_{i}-x_{j}\right\| \\
& \leq\left\|P_{m} x_{i}-P_{m} x_{j}\right\|+\left\|\left(\mathrm{id}_{T}-P_{m}\right) x_{i}\right\|+\left\|\left(\mathrm{id}_{T}-P_{m}\right) x_{j}\right\| \leq 3 \delta,
\end{aligned}
$$

which is clearly impossible. But then for $L:\left[t_{i}\right]_{i=1}^{m} \rightarrow\left(\mathrm{id}_{T}-P_{m}\right) X_{j_{0}}, x \mapsto$ $\left(\operatorname{id}_{T}-P_{m}\right) s_{j_{0}} x$, we would have $\|L\|\left\|L^{-1}\right\| \leq \delta^{-1}\left\|s_{j_{0}}\right\|\left\|r_{j_{0}}\right\|$, contradicting our choice of $m$.

We do not know whether there are bounded sequences $\left(r_{i}\right)$ and $\left(s_{i}\right)$ in $\mathcal{B}(T)$ so that $r_{i} s_{j}=\delta_{i, j} \operatorname{id}_{T}(i, j \in \mathbb{N})$. In fact, we do not even know whether the sequences $\left(r_{i}\right)$ and $\left(s_{i}\right)$ can be chosen so that the averages $n^{-1} \sum_{i=1}^{n} r_{i} s_{i}$ be uniformly bounded. For this reason, we will follow here a path similar to that of $[\mathrm{Bl}$, Section 5]. We start with the following technical result.

Lemma 5.1. Let $\mathcal{A}$ be a Banach algebra, let $e \in \mathcal{A}$ be an idempotent and let $\left(r_{i}\right)$ and $\left(s_{i}\right)$ be sequences in $\mathcal{A}$ such that $r_{i} s_{j}=\delta_{i, j} e(i, j \in \mathbb{N})$. Suppose, in addition, that $s_{i} e=s_{i}$ and $e r_{i}=r_{i}(i \in \mathbb{N})$. Let $\mathfrak{X}$ be a Banach $\mathcal{A}$-bimodule and let $D: \mathcal{A} \rightarrow \mathfrak{X}^{\prime}$ be a derivation. Then, for every $\xi \in \mathfrak{X}$ and every $n \in \mathbb{N}$, we have

$$
\left\langle e \xi e, \varphi_{2^{n}}\right\rangle=\left\langle e \xi e, r_{1} \cdot D\left(s_{1}\right)\right\rangle+\sum_{i=0}^{n-1} 2^{-i-1}\left\langle\sum_{j=1}^{2^{i}} s_{j} \xi r_{j}, \gamma_{i}\right\rangle,
$$

where $\gamma_{i}:=\left(\sum_{j=1}^{2^{i}} s_{j} r_{2^{i}+j}\right) \cdot D\left(\sum_{j=1}^{2^{i}} s_{2^{i}+j} r_{j}\right) \quad(i \in \mathbb{N} \cup\{0\})$.

Proof. The proof is by induction on $n$. The case $n=1$ is easily verified. Suppose the identity has been established for some $n$. Then note that 


$$
\begin{aligned}
\left\langle\sum_{j=1}^{2^{n}} s_{j} \xi r_{j}, \gamma_{n}\right\rangle & =\left\langle\sum_{j=1}^{2^{n}} s_{j} \xi r_{j},\left(\sum_{j=1}^{2^{n}} s_{j} r_{2^{n}+j}\right) \cdot D\left(\sum_{j=1}^{2^{n}} s_{2^{n}+j} r_{j}\right)\right\rangle \\
& =\left\langle\sum_{j=1}^{2^{n}} s_{j} \xi r_{j}, \sum_{j=1}^{2^{n}}\left(s_{j} r_{2^{n}+j}\right) \cdot D\left(s_{2^{n}+j} r_{j}\right)\right\rangle \\
& =\sum_{j=1}^{2^{n}}\left\langle s_{j} \xi e, r_{2^{n}+j} \cdot D\left(s_{2^{n}+j} r_{j}\right)\right\rangle \\
& =\sum_{j=1}^{2^{n}}\left\langle e \xi e, r_{2^{n}+j} \cdot D\left(s_{2^{n}+j}\right)\right\rangle+\sum_{j=1}^{2^{n}}\left\langle e \xi e, D\left(r_{j}\right) \cdot s_{j}\right\rangle \\
& =\sum_{j=2^{n}+1}^{2^{n+1}}\left\langle e \xi e, r_{j} \cdot D\left(s_{j}\right)\right\rangle-\sum_{j=1}^{2^{n}}\left\langle e \xi e, r_{j} \cdot D\left(s_{j}\right)\right\rangle .
\end{aligned}
$$

Combining this last with the induction hypothesis, we obtain

$$
\begin{aligned}
\left\langle e \xi e, r_{1} \cdot D\left(s_{1}\right)\right\rangle+ & \sum_{i=0}^{n} 2^{-i-1}\left\langle\sum_{j=1}^{2^{i}} s_{j} \xi r_{j}, \gamma_{i}\right\rangle \\
= & \left\langle e \xi e, \varphi_{2^{n}}\right\rangle+2^{-n-1}\left\langle\sum_{j=1}^{2^{n}} s_{j} \xi r_{j}, \gamma_{n}\right\rangle \\
= & 2^{-n} \sum_{j=1}^{2^{n}}\left\langle e \xi e, r_{j} \cdot D\left(s_{j}\right)\right\rangle-2^{-n-1} \sum_{j=1}^{2^{n}}\left\langle e \xi e, r_{j} \cdot D\left(s_{j}\right)\right\rangle \\
& +2^{-n-1} \sum_{j=2^{n}+1}^{2^{n+1}}\left\langle e \xi e, r_{j} \cdot D\left(s_{j}\right)\right\rangle \\
= & \left\langle e \xi e, \varphi_{2^{n+1}}\right\rangle,
\end{aligned}
$$

and so the identity holds for $n+1$ too.

COROLlary 5.2. Let $X$ be a Banach space such that there exist sequences $\left(r_{i}\right)$ and $\left(s_{i}\right)$ in $\mathcal{B}(X)$ satisfying $r_{i} s_{j}=\delta_{i, j} \operatorname{id}_{X}(i, j \in \mathbb{N})$. Let

$$
\Gamma_{n}:=\left\|\sum_{j=1}^{n} s_{j} r_{n+j}\right\|\left\|\sum_{j=1}^{n} s_{n+j} r_{j}\right\| \quad(n \in \mathbb{N}) .
$$

If

$$
\left\|\Delta_{2^{i}}(w)\right\|=o\left(\sqrt{2^{i}}\right) \quad \text { and } \quad \sum_{i=0}^{\infty} \frac{\Gamma_{2^{i}}\left\|\Delta_{2^{i}}(w)\right\|}{2^{i}}<\infty \quad(w \in \mathcal{B}(X)),
$$

then $\mathcal{B}(X)$ is weakly amenable. 
Proof. Let $D: \mathcal{B}(X) \rightarrow \mathcal{B}(X)^{\prime}$ be a continuous derivation. By Lemma 5.1, $\left|\left\langle w, \varphi_{2^{n}}\right\rangle\right| \leq\|D\|\left\|r_{1}\right\|\left\|s_{1}\right\|\|w\|+\|D\| \sum_{i=0}^{\infty} 2^{-i-1} \Gamma_{2^{i}}\left\|\Delta_{2^{i}}(w)\right\|(w \in \mathcal{B}(X))$. Thus, $\left(\varphi_{2^{n}}\right)$ is a bounded sequence.

On the other hand,

$$
\left|\left\langle\Delta_{2^{i}}(v), D\left(\Delta_{2^{i}}(w)\right)\right\rangle\right| \leq\|D\|\left\|\Delta_{2^{i}}(v)\right\|\left\|\Delta_{2^{i}}(w)\right\|=o\left(2^{i}\right) \quad(v, w \in \mathcal{B}(X)) .
$$

To finish the proof one just needs to apply Theorem 3.4 .

COROllary 5.3. The algebra $\mathcal{B}(T)$ is weakly amenable.

Before giving the proof, we need to recall a few basic facts about $T$.

As above, let $\left(t_{i}\right)$ be the unit vector basis of $T$. Then for every $\sum_{i} \alpha_{i} t_{i} \in T$ and every strictly increasing sequence $\left(n_{i}\right)$ of positive integers,

$$
\left\|\sum_{i} \alpha_{i} t_{i}\right\| \leq\left\|\sum_{i} \alpha_{i} t_{n_{i}}\right\|
$$

In the opposite direction, there exists a constant $K>0$ such that

$$
\left\|\sum_{i} \alpha_{i} t_{2^{i}}\right\| \leq K\left\|\sum_{i} \alpha_{i} t_{i}\right\| \quad\left(\sum_{i} \alpha_{i} t_{i} \in T\right) .
$$

If $L$ and $R$ are the left and right shift operators, respectively, with respect to $\left(t_{i}\right)$ then $\left\|L^{n}\right\|=1$ and $\left\|R^{n}\right\|=O\left(n^{3}\right)(n \in \mathbb{N})([\mathrm{Bl}$, Lemma 5.3]).

We will also need the following.

Lemma 5.4 ([CS, Proposition V.12]). For any $n \in \mathbb{N}$, let $\left\{I_{n}, I_{n+1}, \ldots\right.$, $\left.I_{n 2^{n}}\right\}$ be a partition of $\mathbb{N} \cap\left[n, \infty\left[\right.\right.$. Set $X_{j}=\left[t_{k}: k \in I_{j}\right]\left(n \leq j \leq n 2^{n}\right)$. Then there exists a constant $M$, independent of $n$ and the partition chosen, such that $\|I\|\left\|I^{-1}\right\| \leq M$, where $I$ denotes the formal identity map from $\left[t_{k}\right]_{k=n}^{\infty}$ to $\left(\oplus_{n \leq j \leq n 2^{n}} X_{j}\right)_{\ell_{1}}$.

Proof of Corollary 5.3. For each $n \in \mathbb{N}$, define $E_{n} \subset \mathbb{N}$ by $E_{n}:=\left\{2^{k}+n:\right.$ $k>\rho(n)\}$, where $\rho(n)=\log _{2} n$, and let $X_{n}:=\left[t_{i}: i \in E_{n}\right]$. Note that $X_{n} \cap X_{m}=\{0\}$ whenever $n \neq m$. Indeed, it suffices to see that $E_{n} \cap E_{m}=\emptyset$ whenever $n \neq m$. For this, suppose towards a contradiction that there are $i>\rho(n)$ and $j>\rho(m)$ so that $2^{i}+n=2^{j}+m$. As $m \neq n$, we must have $i \neq j$. Without loss of generality, suppose $i<j$. Then $\rho(n)<i \Rightarrow n<2^{i} \Rightarrow$ $2^{i}+n<2^{i+1}<2^{j}+m$, a contradiction.

Next, for every $n \in \mathbb{N}$, define $s_{n} \in \mathcal{B}(T)$ by $s_{n}\left(t_{i}\right):=t_{2^{\rho(n)+i-1}+n}(i \in \mathbb{N})$, and let $r_{n} \in \mathcal{B}(T)$ be so that $r_{n} s_{n}=\mathrm{id}_{T}$ and $s_{n} r_{n}\left(=: \pi_{n}\right)$ is the natural projection onto $X_{n}$. From (7) and (8) one sees that, for every $x=\sum_{i} \alpha_{i} t_{i} \in T$,

$$
\left\|s_{n}(x)\right\| \leq\left\|\sum_{i} \alpha_{i} t_{2^{\rho(n)+i}}\right\| \leq K\left\|\sum_{i} \alpha_{i} t_{\rho(n)+i}\right\| \leq K\left\|R^{\rho(n)}\right\|\|x\|,
$$

so $\left\|s_{n}\right\|=O\left(\rho(n)^{3}\right)$. 
Let $w \in \mathcal{B}(T), x \in T$ and $n \in \mathbb{N}$ be arbitrary. Then

$$
\begin{aligned}
\left\|\Delta_{2^{n}}(w)(x)\right\| & =\left\|\sum_{k=1}^{2^{n}} s_{k} w r_{k} L^{n} R^{n} x_{k}\right\| \leq\left(\max _{k}\left\|s_{k}\right\|\left\|r_{k}\right\|\right)\|w\| \sum_{k=1}^{2^{n}}\left\|R^{n} x_{k}\right\| \\
& \leq\left(\max _{k}\left\|s_{k}\right\|\left\|r_{k}\right\|\right)\|w\| M\left\|R^{n}\right\|\|x\|,
\end{aligned}
$$

where $x_{k}=\pi_{k} x(k \in \mathbb{N})$ and the second inequality follows from Lemma 5.4 Combining this last with our estimates for $\left\|s_{n}\right\|$ and $\left\|R^{n}\right\|$ one readily gets

$$
\left\|\Delta_{2^{n}}(w)\right\|=\left(\max _{k}\left\|s_{k}\right\|\left\|r_{k}\right\|\right)\|w\| M\left\|R^{n}\right\|=O\left(n^{6}\right) .
$$

Similarly, one verifies that, for $n \in \mathbb{N}$,

$$
\begin{aligned}
& \left\|\sum_{k=1}^{2^{n}} s_{k} r_{2^{n}+k}\right\| \leq M \max _{k}\left\|s_{k} r_{2^{n}+k}\right\|=O\left(n^{3}\right), \\
& \left\|\sum_{k=1}^{2^{n}} s_{2^{n}+k} r_{k}\right\| \leq M\left\|R^{n}\right\| \max _{k}\left\|s_{2^{n}+k} r_{k}\right\|=O\left(n^{6}\right) .
\end{aligned}
$$

The desired result then follows as a consequence of Corollary 5.2

6. Direct sums. In this final section we present some results on weak amenability of $\mathcal{B}(X)$ in the case where $X$ is a finite direct sum of Banach spaces with direct sum decompositions of the kind considered in Section 4. Strictly speaking, these results should not be seen as results on direct sums but rather as further applications of Theorem 3.4. The main result of the section is the following.

Proposition 6.1. Let $X_{1}$ and $X_{2}$ be Banach spaces with decompositions $\bigoplus_{i=0}^{\infty} X_{1, i}$ and $\bigoplus_{i=0}^{\infty} X_{2, i}$, respectively, such that $\sup _{i} d\left(X_{1}, X_{1, i}\right)<\infty$ and $\sup _{i} d\left(X_{2}, X_{2, i}\right)<\infty$. Moreover, suppose there are $1<s \leq r<\infty$ so that at least one of the following holds:

(1) $\bigoplus_{i=0}^{\infty} X_{1, i}$ satisfies a lower $r$-estimate and an upper s-estimate, and $\bigoplus_{i=0}^{\infty} X_{2, i}$ satisfies an upper $r$-estimate.

(2) $\bigoplus_{i=0}^{\infty} X_{1, i}$ satisfies a lower $r$-estimate and an upper s-estimate, and $\bigoplus_{i=0}^{\infty} X_{2, i}$ satisfies a lower s-estimate.

If $\mathcal{B}\left(X_{1}\right)$ and $\mathcal{B}\left(X_{2}\right)$ are weakly amenable then so is $\mathcal{B}\left(X_{1} \oplus X_{2}\right)$.

Proof. Let $\pi_{i}: X_{1} \oplus X_{2} \rightarrow X_{i}$ (resp. $\imath_{i}: X_{i} \rightarrow X_{1} \oplus X_{2}$ ), $i=1,2$, stand for the canonical $i$ th coordinate projection (resp. embedding), and for every $w \in \mathcal{B}\left(X_{1} \oplus X_{2}\right)$, let $w_{i j}:=\pi_{i} w \imath_{j}(1 \leq i, j \leq 2)$. Also, let $\pi_{i, j}: X_{i} \rightarrow X_{i, j}$ (resp. $\imath_{i, j}: X_{i, j} \rightarrow X_{i}$ ), $i \in\{1,2\}, j \in \mathbb{N}$, denote the corresponding canonical projection (resp. embedding) with respect to the decomposition $\bigoplus_{j=0}^{\infty} X_{i, j}$.

Choose bounded sequences $\left(r_{i, j}\right)_{j \in \mathbb{N}}$ and $\left(s_{i, j}\right)_{j \in \mathbb{N}}$ in $\mathcal{B}\left(X_{i}\right)$ so that $r_{i, j} s_{i, k}$ $=\delta_{j, k} \pi_{i} \imath_{i}$ and $s_{i, j} r_{i, j}=\imath_{i, j} \pi_{i, j}(j, k \in \mathbb{N}, i=1,2)$, and define $r_{j}:=\imath_{1} r_{1, j} \pi_{1}+$ 
$\imath_{2} r_{2, j} \pi_{2}$ and $s_{j}:=\imath_{1} s_{1, j} \pi_{1}+\imath_{2} s_{2, j} \pi_{2}(j \in \mathbb{N})$. Note that the sequences $\left(r_{j}\right)$ and $\left(s_{j}\right)$, defined in this way, commute with $\imath_{i} \pi_{i}(i=1,2)$.

Suppose (1) holds, and to fix ideas, suppose that

$$
c_{1}^{-1}\left(\sum_{j}\left\|x_{1, j}\right\|^{r}\right)^{1 / r} \leq\left\|\sum_{j} x_{1, j}\right\| \leq C_{1}\left(\sum_{j}\left\|x_{1, j}\right\|^{s}\right)^{1 / s}
$$

and

$$
\left\|\sum_{j} x_{2, j}\right\| \leq C_{2}\left(\sum_{j}\left\|x_{2, j}\right\|^{r}\right)^{1 / r}
$$

whenever $\left(x_{1, j}\right)$ and $\left(x_{2, j}\right)$ are finite sequences in $X_{1}$ and $X_{2}$, respectively, so that $x_{1, j} \in X_{1, j}$ and $x_{2, j} \in X_{2, j}$ for every $j$.

Then the decomposition $\bigoplus_{j}\left(X_{1, j} \oplus X_{2, j}\right)$ of $X_{1} \oplus X_{2}$ satisfies an upper $s$-estimate. Indeed,

$$
\begin{aligned}
\left\|\sum_{j}\left(x_{1, j}, x_{2, j}\right)\right\| & =\left\|\left(\sum_{j} x_{1, j}, \sum_{j} x_{2, j}\right)\right\| \leq\left\|\imath_{1}\right\|\left\|\sum_{j} x_{1, j}\right\|+\left\|\imath_{2}\right\|\left\|\sum_{j} x_{2, j}\right\| \\
& \leq C_{1}\left\|\imath_{1}\right\|\left(\sum_{j}\left\|x_{1, j}\right\|^{s}\right)^{1 / s}+C_{2}\left\|\imath_{2}\right\|\left(\sum_{j}\left\|x_{2, j}\right\|^{r}\right)^{1 / r} \\
& \leq\left(C_{1}\left\|\imath_{1}\right\|\left\|\pi_{1}\right\|+C_{2}\left\|\imath_{2}\right\|\left\|\pi_{2}\right\|\right)\left(\sum_{j}\left\|\left(x_{1, j}, x_{2, j}\right)\right\|^{s}\right)^{1 / s} .
\end{aligned}
$$

Thus, to establish the desired result, it will be enough, by Corollary 4.2 , to show that for every pair $u, v \in \mathcal{B}\left(X_{1} \oplus X_{2}\right)$ and for every continuous derivation $D: \mathcal{B}\left(X_{1} \oplus X_{2}\right) \rightarrow \mathcal{B}\left(X_{1} \oplus X_{2}\right)^{\prime}$,

$$
n^{-1}\left\langle\Delta_{n}(u), D\left(\Delta_{n}(v)\right)\right\rangle \rightarrow 0,
$$

where $\Delta_{n}(w):=\sum_{k=1}^{n} s_{k} w r_{k}\left(w \in \mathcal{B}\left(X_{1} \oplus X_{2}\right)\right)$. One easily sees that

$$
\langle u, D(v)\rangle=\sum_{i, j}\left\langle\imath_{i} u_{i j} \pi_{j}, D\left(\imath_{j} v_{j i} \pi_{i}\right)\right\rangle,
$$

and in turn,

$$
\begin{aligned}
\left\langle\Delta_{n}(u), D\left(\Delta_{n}(v)\right)\right\rangle & =\sum_{k}\left\langle s_{k} u r_{k}, D\left(s_{k} v r_{k}\right)\right\rangle \\
& =\sum_{k} \sum_{i, j}\left\langle\imath_{i} \pi_{i} s_{k} u r_{k} \imath_{j} \pi_{j}, D\left(\imath_{j} \pi_{j} s_{k} v r_{k} \imath_{i} \pi_{i}\right)\right\rangle \\
& =\sum_{i, j}\left\langle\Delta_{n}\left(\imath_{i} u_{i j} \pi_{j}\right), D\left(\Delta_{n}\left(\imath_{j} v_{j i} \pi_{i}\right)\right)\right\rangle .
\end{aligned}
$$

So, in order to establish (9), it suffices to show that

$$
n^{-1}\left\langle\Delta_{n}\left(\imath_{i} u_{i j} \pi_{j}\right), D\left(\Delta_{n}\left(\imath_{j} v_{j i} \pi_{i}\right)\right)\right\rangle \rightarrow 0 \quad(1 \leq i, j \leq 2) .
$$


Next note that

$$
\begin{aligned}
\left\langle\Delta_{n}\left(\imath_{i} u_{i i} \pi_{i}\right), D\left(\Delta_{n}\left(\imath_{i} v_{i i} \pi_{i}\right)\right)\right\rangle & =\left\langle\imath_{i} \Delta_{i, n}\left(u_{i i}\right) \pi_{i}, D\left(\imath_{i} \Delta_{i, n}\left(v_{i i}\right) \pi_{i}\right)\right\rangle \\
& =\left\langle\Delta_{i, n}\left(u_{i i}\right), D_{i i}\left(\Delta_{i, n}\left(v_{i i}\right)\right)\right\rangle,
\end{aligned}
$$

where $\Delta_{i, n}: \mathcal{B}\left(X_{i}\right) \rightarrow \mathcal{B}\left(X_{i}\right), w \mapsto \sum_{k=1}^{n} s_{i, k} w r_{i, k}$, and $D_{i i}: \mathcal{B}\left(X_{i}\right) \rightarrow \mathcal{B}\left(X_{i}\right)^{\prime}$ is defined by $\left\langle u, D_{i i}(v)\right\rangle=\left\langle u, \pi_{i} \cdot D\left(\imath_{i} v \pi_{i}\right) \cdot \imath_{i}\right\rangle(i=1,2)$. One easily verifies that $D_{i i}(i=1,2)$ is a derivation. That $n^{-1}\left\langle\Delta_{n}\left(\imath_{i} u_{i i} \pi_{i}\right), D\left(\Delta_{n}\left(\imath_{i} v_{i i} \pi_{i}\right)\right)\right\rangle \rightarrow 0$ $(i=1,2)$ now follows immediately from Corollary 4.2 , since $\bigoplus_{k=0}^{\infty} X_{i, k}$ satisfies an upper $s$-estimate and $\mathcal{B}\left(X_{i}\right)$ is weakly amenable.

As for the other two cases, we argue as follows. Let $\left(x_{1}, x_{2}\right) \in X_{1} \oplus X_{2}$ and $w \in \mathcal{B}\left(X_{1} \oplus X_{2}\right)$ be arbitrary. First note that

$$
\begin{aligned}
\left\|\Delta_{n}\left(\imath_{1} w_{12} \pi_{2}\right)\left(x_{1}, x_{2}\right)\right\| & \\
& \leq\left\|\sum_{k} \imath_{1} s_{1, k} w_{12} r_{2, k} x_{2}\right\| \leq C_{1}\left\|\imath_{1}\right\|\left(\sum_{k}\left\|s_{1, k} w_{12} r_{2, k} x_{2, k}\right\|^{s}\right)^{1 / s} \\
& \leq C_{1} n^{1 / s}\left\|\imath_{1}\right\|\left(\sup _{k}\left\|s_{1, k}\right\|\left\|r_{2, k}\right\|\right)\left\|w_{12}\right\| \sup _{k}\left\|x_{2, k}\right\| \\
& \leq 2 C_{1} C n^{1 / s}\left\|\imath_{1}\right\|\left\|\pi_{2}\right\|\left(\sup _{k}\left\|s_{1, k}\right\|\left\|r_{2, k}\right\|\right)\left\|w_{12}\right\|\left\|\left(x_{1}, x_{2}\right)\right\|,
\end{aligned}
$$

where $C$ is the constant of the decomposition $\bigoplus_{k=0}^{\infty} X_{2, k}$. So

$$
\left\|\Delta_{n}\left(\imath_{1} w_{12} \pi_{2}\right)\right\| \leq K n^{1 / s}\left\|w_{12}\right\|
$$

for some constant $K$ independent of $w$ and $n$. Second,

$$
\begin{aligned}
\left\|\Delta_{n}\left(\imath_{2} w_{21} \pi_{1}\right)\left(x_{1}, x_{2}\right)\right\| & \\
\leq & \leq \sum_{k} \imath_{2} s_{2, k} w_{21} r_{1, k} x_{1}\left\|\leq C_{2}\right\| \imath_{2} \|\left(\sum_{k}\left\|s_{2, k} w_{21} r_{1, k} x_{1, k}\right\|^{r}\right)^{1 / r} \\
& \leq C_{2}\left\|\imath_{2}\right\|\left(\sup _{k}\left\|s_{2, k}\right\|\left\|r_{1, k}\right\|\right)\left\|w_{21}\right\|\left(\sum_{k}\left\|x_{1, k}\right\|^{r}\right)^{1 / r} \\
& \leq C_{2} c_{1}\left\|\imath_{2}\right\|\left\|\pi_{1}\right\|\left(\sup _{k}\left\|s_{2, k}\right\|\left\|r_{1, k}\right\|\right)\left\|w_{21}\right\|\left\|\left(x_{1}, x_{2}\right)\right\| .
\end{aligned}
$$

So,

$$
\left\|\Delta_{n}\left(\imath_{2} w_{21} \pi_{1}\right)\right\| \leq L\left\|w_{21}\right\|,
$$

where $L$ is a constant independent of $w$ and $n$.

Thus, if $i \neq j$ then, combining the above estimates, one obtains

$$
\left|\left\langle\Delta_{n}\left(\imath_{i} u_{i j} \pi_{j}\right), D\left(\Delta_{n}\left(\imath_{j} v_{j i} \pi_{i}\right)\right)\right\rangle\right| \leq K L n^{1 / s}\|D\|\left\|u_{i j}\right\|\left\|v_{j i}\right\|=o(n),
$$

as needed. This concludes the proof in the case where (1) is satisfied.

If (2) is satisfied the proof is very similar, so we will not give it in full detail. In this case, one can show that $\bigoplus_{j}\left(X_{1, j} \oplus X_{2, j}\right)$ satisfies a 
lower $r$-estimate. Then, taking into account this last and the fact that $\mathcal{B}\left(X_{i}\right)$ is weakly amenable $(i=1,2)$, one can deduce, exactly as above, that $n^{-1}\left\langle\Delta_{n}\left(\imath_{i} u_{i i} \pi_{i}\right), D\left(\Delta_{n}\left(\imath_{i} v_{i i} \pi_{i}\right)\right)\right\rangle \rightarrow 0$ as $n \rightarrow \infty(i=1,2)$. Finally, one can find constants $K_{1}$ and $L_{1}$ so that, for every $w \in \mathcal{B}\left(X_{1} \oplus X_{2}\right)$,

$$
\left\|\Delta_{n}\left(\imath_{1} w_{12} \pi_{2}\right)\right\| \leq K_{1}\left\|w_{12}\right\| \quad \text { and } \quad\left\|\Delta_{n}\left(\imath_{2} w_{21} \pi_{1}\right)\right\| \leq L_{1} n^{(r-1) / r}\left\|w_{21}\right\| .
$$

That $n^{-1}\left\langle\Delta_{n}\left(\imath_{i} u_{i j} \pi_{j}\right), D\left(\Delta_{n}\left(\imath_{j} v_{j i} \pi_{i}\right)\right)\right\rangle \rightarrow 0$ as $n \rightarrow \infty(1 \leq i \neq j \leq 2)$ follows readily from these last two inequalities.

As a consequence of Proposition 6.1 we have the following.

Corollary 6.2. Let $E_{1}, \ldots, E_{n}$ be Banach spaces so that at least one of the following holds:

(1) For every $1 \leq i \leq n$ there exists $1 \leq p_{i}<\infty$ so that $E_{i} \simeq \ell_{p_{i}}\left(E_{i}\right)$.

(2) For every $1 \leq i \leq n$ there exists $1<p_{i} \leq \infty$ so that $E_{i} \simeq \ell_{p_{i}}\left(E_{i}\right)$. Then $\mathcal{B}\left(E_{1} \oplus \cdots \oplus E_{n}\right)$ is weakly amenable.

Proof. It will be enough to show that if either $1 \leq p_{n}<p_{n-1}<\cdots<$ $p_{1}<\infty$ or $1<p_{1}<\cdots<p_{n-1}<p_{n} \leq \infty$ then $\mathcal{B}\left(\ell_{p_{1}}\left(E_{1}\right) \oplus \cdots \oplus \ell_{p_{n}}\left(E_{n}\right)\right)$ is weakly amenable. Note that if $p_{i}=p_{j}$ for some $1 \leq i \neq j \leq n$ then $\ell_{p_{i}}\left(E_{i}\right) \oplus \ell_{p_{j}}\left(E_{j}\right) \simeq \ell_{p_{i}}\left(E_{i} \oplus E_{j}\right)$.

Let us start by considering the case where $1 \leq p_{n}<p_{n-1}<\cdots<p_{1}$ $<\infty$. We argue by induction on $n$. By Proposition 3.2, the result is true for $n=1$. Let us suppose the result has been established for $n=k$. We then prove it for $n=k+1$.

Set $X_{1}=\ell_{p_{1}}\left(E_{1}\right) \oplus \cdots \oplus \ell_{p_{k}}\left(E_{k}\right)$ and $X_{2}=\ell_{p_{k+1}}\left(E_{k+1}\right)$. Moreover, let $\left\{N_{i}: i \in \mathbb{N}\right\}$ be a partition of $\mathbb{N}$ into infinite subsets, and let $X_{l, j}=\left\{\left(e_{i}\right) \in\right.$ $\ell_{p_{l}}\left(E_{l}\right): e_{i}=0$ if $\left.i \notin N_{j}\right\}, 1 \leq l \leq n+1$. Then $X_{1}=\oplus_{l=1}^{k}\left(\bigoplus_{j=1}^{\infty} X_{l, j}\right)=$ $\bigoplus_{j=1}^{\infty}\left(\oplus_{l=1}^{k} X_{l, j}\right)$ and $\oplus_{l=1}^{k} X_{l, j} \cong X_{1}(j \in \mathbb{N})$. This last decomposition of $X_{1}$ satisfies a lower $p_{1}$-estimate and an upper $p_{k}$-estimate. Indeed, let $\pi_{l}$ : $X_{1} \rightarrow \ell_{p_{l}}\left(E_{l}\right)$ (resp. $\left.\imath_{l}: \ell_{p_{l}}\left(E_{l}\right) \rightarrow X_{1}\right), 1 \leq l \leq k$, denote the canonical $l$ th coordinate projection (resp. embedding). Then

$$
\begin{aligned}
& \left(\sum_{j}\left\|\left(x_{1, j}, \ldots, x_{k, j}\right)\right\|^{p_{1}}\right)^{1 / p_{1}} \\
& \quad \leq\left(\sum_{j}\left(\sum_{l=1}^{k}\left\|\imath_{l}\right\|\left\|x_{l, j}\right\|\right)^{p_{1}}\right)^{1 / p_{1}} \leq \sum_{l=1}^{k}\left\|\imath_{l}\right\|\left(\sum_{j}\left\|x_{l, j}\right\|^{p_{1}}\right)^{1 / p_{1}} \\
& \quad=\sum_{l=1}^{k}\left\|\imath_{l}\right\|\left\|\sum_{j} x_{l, j}\right\| \leq M\left\|\sum_{j}\left(x_{1, j}, \ldots, x_{k, j}\right)\right\|
\end{aligned}
$$


and

$$
\begin{aligned}
\left\|\sum_{j}\left(x_{1, j}, \ldots, x_{k, j}\right)\right\| \\
\leq\left\|\left(\sum_{j} x_{1, j}, \ldots, \sum_{j} x_{k, j}\right)\right\| \leq \sum_{l=1}^{k}\left\|\imath_{l}\right\|\left(\sum_{j}\left\|x_{l, j}\right\|^{p_{l}}\right)^{1 / p_{l}} \\
\leq M\left(\sum_{j}\left\|\left(x_{1, j}, \ldots, x_{k, j}\right)\right\|^{p_{k}}\right)^{1 / p_{k}}
\end{aligned}
$$

where $M=\sum_{l=1}^{k}\left\|\imath_{l}\right\|\left\|\pi_{l}\right\|$. Also, it is easy to see that $\bigoplus_{j=1}^{\infty} X_{k+1, j}$ satisfies a lower $p_{k}$-estimate, so condition (2) of Proposition 6.1 holds. As $\mathcal{B}\left(\ell_{p_{k+1}}\left(E_{k+1}\right)\right)$ is weakly amenable and, by the induction hypothesis, $\mathcal{B}\left(X_{1}\right)$ is weakly amenable too, we can apply Proposition 6.1 to conclude that $\mathcal{B}\left(X_{1} \oplus \ell_{p_{k+1}}\left(E_{k+1}\right)\right)$ is weakly amenable.

If $1<p_{1}<\cdots<p_{n} \leq \infty$ then one can argue in almost the same way as in the previous paragraph. We only need to note that, in the induction argument, if one defines $X_{1}, X_{2}, \bigoplus_{j} X_{1, j}$ and $\bigoplus_{j} X_{2, j}$ exactly as we did before, then $\bigoplus_{j} X_{1, j}$ satisfies a lower $p_{k}$-estimate and an upper $p_{1}$-estimate, and $\bigoplus_{j} X_{2, j}$ satisfies an upper $p_{k}$-estimate, i.e., (1) of Proposition 6.1 is satisfied instead of (2). The rest of the argument remains the same.

Corollary 6.3. The algebra $\mathcal{B}\left(\ell_{p_{1}} \oplus \cdots \oplus \ell_{p_{n}}\right)$, where $1 \leq p_{1}, \ldots, p_{n}$ $<\infty$ (resp. $\left.1<p_{1}, \ldots, p_{n} \leq \infty\right)$, is weakly amenable.

Proof. This is an immediate consequence of the previous corollary.

REMARK 6.4. The strict inequality in the hypotheses of Corollary 6.3 is not an essential requirement. However, the extreme cases need to be handled differently. Let us show, for instance, that $\mathcal{B}\left(c_{0} \oplus \ell_{1}\right)$ is weakly amenable. For this, let $\mathcal{C}(X)$ denote the quotient $\mathcal{B}(X) / \mathcal{A}(X)$. By Pitt's theorem, $\mathcal{C}\left(c_{0} \oplus \ell_{1}\right)$ can be represented as a unital algebra of $2 \times 2$ upper triangular matrices, with diagonal entries coming from the unital Banach algebras $\mathcal{C}\left(c_{0}\right)$ and $\mathcal{C}\left(\ell_{1}\right)$. As $\mathcal{C}\left(c_{0}\right)$ and $\mathcal{C}\left(\ell_{1}\right)$ are weakly amenable, so is $\mathcal{C}\left(c_{0} \oplus \ell_{1}\right)$, by Ly, Proposition 2.11]. Then, as $\mathcal{A}\left(c_{0} \oplus \ell_{1}\right)$ is weakly amenable ([B12, Corollary 3.8]) and $\mathcal{C}\left(c_{0} \oplus \ell_{1}\right)$ is weakly amenable, $\mathcal{B}\left(c_{0} \oplus \ell_{1}\right)$ is weakly amenable too (see [LW] Proposition 5.5]).

Unfortunately, the conditions of Corollary 6.1 are not conditions that one can verify separately for each summand (as in [B12, Theorem 3.6], for instance), and we have not been able to make much progress in this direction. We thus finish this note with the following question.

Suppose $\mathcal{B}(X)$ and $\mathcal{B}(Y)$ are weakly amenable. Is $\mathcal{B}(X \oplus Y)$ weakly amenable? 


\section{References}

[BCD] W. G. Bade, P. C. Curtis and H. G. Dales, Amenability and weak amenability for Beurling and Lipschitz algebras, Proc. London Math. Soc. (3) 55 (1987), 359-377.

[Be] B. Beauzamy, Introduction to Banach Spaces and Their Geometry, North-Holland Math. Stud. 68, North-Holland, Amsterdam, 1982.

[Bl] A. Blanco, Weak amenability of $\mathcal{A}(E)$ and the geometry of $E$, J. London Math. Soc. (2) 66 (2002), 721-740.

[Bl2] -, On the weak amenability of $\mathcal{A}(X)$ and its relation with the approximation property, J. Funct. Anal. 203 (2003), 1-26.

[BG] A. Blanco and N. Grønbæk, Cohomology of Banach algebras of operators and geometry of Banach spaces, in: Function Spaces, Contemp. Math. 435, Amer. Math. Soc., Providence, RI, 2007, 57-80.

[BG1] - - - Amenability of algebras of approximable operators, Israel J. Math. 171 (2009), 127-156.

[CS] P. G. Casazza and T. J. Shura, Tsirelson's Space, Lecture Notes in Math. 1363, Springer, Berlin, 1989.

[Da] H. G. Dales, Banach Algebras and Automatic Continuity, London Math. Soc. Monogr. 24, Clarendon Press, Oxford, 2000.

[DGG] H. G. Dales, F. Ghahramani and N. Grønbæk, Derivations into iterated duals of Banach algebras, Studia Math. 128 (1998), 19-54.

[D] J. Diestel, Sequences and Series in Banach Spaces, Grad. Texts in Math. 92, Springer, New York, 1984.

[G] N. Grønbæk, Various notions of amenability, a survey of problems, in: Banach Algebras '97 (Blaubeuren), de Gruyter, Berlin, 1998, 535-547.

[G1] - Bounded Hochschild cohomology of Banach algebras with a matrix-like structure, Trans. Amer. Math. Soc. 358 (2006), 2651-2662.

[H] U. Haagerup, All nuclear $C^{*}$-algebras are amenable, Invent. Math. 74 (1983), 305-319.

[J] B. E. Johnson, Continuity of homomorphisms of algebras of operators, J. London Math. Soc. 42 (1967), 537-541.

[J1] -, Cohomology in Banach algebras, Mem. Amer. Math. Soc. 127 (1972).

[J2] - Derivations from $L^{1}(G)$ into $L^{1}(G)$ and $L^{\infty}(G)$, in: Harmonic Analysis (Luxembourg, 1987), Lecture Notes in Math. 1359, Springer, Berlin, 1988, 191-198.

[J3] -, Weak amenability of group algebras, Bull. London Math. Soc. 23 (1991), 281-284.

[La] N. J. Laustsen, Commutators of operators on Banach spaces, J. Operator Theory 48 (2002), suppl., 503-514.

[La1] -, On ringtheoretic (in)finiteness of Banach algebras of operators on Banach spaces, Glasgow Math. J. 45 (2003), 11-19.

[LP] J. Lindenstrauss and A. Pełczyński, Absolutely summing operators in $\mathcal{L}_{p}$-spaces and their applications, Studia Math. 29 (1968), 275-326.

[Ly] Z. A. Lykova, Relative cohomology of Banach algebras, J. Operator Theory 41 (1999), 23-53.

[LW] Z. A. Lykova and M. C. White, Excision in the cohomology of Banach algebras with coefficients in dual bimodules, in: Banach Algebras '97 (Blaubeuren), de Gruyter, Berlin, 1998, 341-361.

[OT] E. Odell and H.-O. Tylli, Weakly compact approximation in Banach spaces, Trans. Amer. Math. Soc. 357 (2005), 1125-1159. 
[R] C. J. Read, Discontinuous derivations on the algebra of bounded operators on a Banach space, J. London Math. Soc. (2) 40 (1989), 305-326.

[R1] - When $E$ and $E[E]$ are isomorphic, in: Geometry of Banach Spaces (Strobl, 1989), London Math. Soc. Lecture Note Ser. 158, Cambridge Univ. Press, Cambridge, 1990, 245-252.

[W] M. Wodzicki, Vanishing of cyclic homology of stable $C^{*}$-algebras, C. R. Acad. Sci. Paris Sér. I Math. 307 (1988), 329-334.
A. Blanco
Department of Pure Mathematics
Queen's University Belfast
Belfast BT7 1NN, UK
E-mail: a.blanco@qub.ac.uk

Received April 27, 2009

Revised version June 16, 2009

(6609) 